\title{
GOODNESS-OF-FIT TESTS DERIVED FROM CHARACTERIZATIONS OF CONTINUOUS DISTRIBUTIONS
}

\author{
DOMINIK SZYNAL \\ Institute of Mathematics, Maria Curie-Sktodowska University \\ pl. M. Curie-Sktodowskiej 1, 20-031 Lublin, Poland \\ E-mail:szynal@poczta.umcs.lublin.pl
}

\begin{abstract}
Starting from characterizations of continuous distributions in terms of the expected values of two functions of record values we construct a family of goodness-of-fit tests calculated from U-statistics.
\end{abstract}

1. Introduction. Characterizations play an important role in many techniques for constructing goodness-of-fit tests. For commonly occurring distributions, for example uniform, exponential and normal, characterizations are given in the literature (cf. Baringhaus and Henze [2], Henze and Meintanis [10, 11], Csörgó et al. [7], Hill and Perez-Abreu [12]). Characterizations of continuous distributions in terms of expected values of order statistics and of record values as presented in Lin [14, Grudzień and Szynal [9], Malinowska et al. [15] were used to construct families of goodness-of-fit tests (cf. Morris and Szynal [16]-[22]). Here we present a family of goodness-of-fit tests using characterizations of continuous distributions via expected values of two functions of record values (cf. Malinowska et al. [15]). They are expressed in terms of $U$-statistics similar to those used in Morris and Szynal [22].

2. Characterization conditions in terms of expected values of two functions of record values. We recall the concepts of the $k$ th upper record values. Let $\left\{X_{n}, n \geq 1\right\}$ be a sequence of i.i.d. random variables with a common cumulative distribution function $F, \bar{F}=1-F$, and probability density $f$. For a fixed integer $k \geq 1$ we define the sequence

2010 Mathematics Subject Classification: Primary 62G10; Secondary 62G30, 62G32.

Key words and phrases: order statistics, record values, uniform, exponential, Rayleigh and normal distribution, characterizations, goodness-of-fit tests, powers.

The paper is in final form and no version of it will be published elsewhere. 
$U_{k}(1), U_{k}(2), \ldots$ of $k$ th upper record times of $\left\{X_{n}, n \geq 1\right\}$ as follows:

$$
\begin{aligned}
& U_{k}(1)=1, \\
& U_{k}(n+1)=\min \left\{j>U_{k}(n): X_{j: j+k-1}>X_{U_{k}(n): U_{k}(n)+k-1}\right\}, \quad n \geq 1 .
\end{aligned}
$$

Write

$$
Y_{n}^{(k)}=X_{U_{k}(n): U_{k}(n)+k-1}, \quad n \geq 1 .
$$

The sequence $\left\{Y_{n}^{(k)}, n \geq 1\right\}$ is called the sequence of $k$ th (upper) record values of the above sequence. For convenience we also take $Y_{0}^{(k)}=0$ and note that

$$
Y_{1}^{(k)}=X_{1: k}=\min \left(X_{1}, \ldots, X_{k}\right)
$$

(Dziubdziela and Kopociński [8]).

We note only the recurrence relations for the cdf of $Y_{n}^{(k)}$ and for its moments that are useful here:

$$
\begin{gathered}
F_{Y_{n}^{(k)}}(x)=F_{Y_{n-1}^{(k)}}(x)-\frac{k^{n-1}}{(n-1) !}[\bar{F}(x)]^{k}[-\log (\bar{F}(x))]^{n-1} \\
E\left(Y_{n}^{(k)}\right)^{\alpha}=E\left(Y_{n-1}^{(k)}\right)^{\alpha}+\alpha \frac{k^{n-1}}{(n-1) !} \int_{-\infty}^{\infty} x^{\alpha-1}(\bar{F}(x))^{k}[-\log (\bar{F}(x))]^{n-1} d x
\end{gathered}
$$

(cf. Grudzień and Szynal [9], Bieniek and Szynal [3], 4]).

Characterizations of continuous distributions via expected values of two functions of record values were first established in Too and Lin [25]. Some extensions were discussed in Grudzień and Szynal [9], Malinowska et al. [15]. Here we discuss and apply characterizations in terms of the expected values of two functions of upper record values.

Let $\left\{X_{n}, n \geq 1\right\}$ be a sequence of random variables distributed as a variate $X$. Assume that $n \geq 1, k \geq 1$ and $s \geq 0, s \neq n$ are given integers and $r \neq 0$ a given real number such that $n+r+1>0$. Then under some conditions (cf. Lin [14], Malinowska et al. [15]) $X \sim F$ and $F$ is continuous iff

$$
\left\{\begin{array}{l}
E\left[-\log \left(\bar{F}\left(Y_{n+1}^{(k)}\right)\right)\right]^{r}=\frac{\Gamma(n+r+1)}{n ! k^{r}} \\
E\left[-\log \left(\bar{F}\left(Y_{s+1}^{(k)}\right)\right)\right]^{r+n-s}=\frac{\Gamma(n+r+1)}{s ! k^{r+n-s}} .
\end{array}\right.
$$

Letting $n=2$ and $s=1$ in (3) we see that $X \sim F$ iff

$$
\left\{\begin{array}{l}
E\left[-\log \left(\bar{F}\left(Y_{3}^{(k)}\right)\right)\right]^{r}=\frac{\Gamma(r+3)}{2 k^{r}}, \\
E\left[-\log \left(\bar{F}\left(Y_{2}^{(k)}\right)\right)\right]^{r+1}=\frac{\Gamma(r+3)}{k^{r+1}} .
\end{array}\right.
$$

for $r>-3$. Thus by (4) we have for $F(x)=1-e^{-x}: X \sim \operatorname{Exp}(1)$ iff

$$
\left\{\begin{array}{l}
E\left[Y_{3}^{(k)}\right]^{r}=\frac{\Gamma(r+3)}{2 k^{r}}, \\
E\left[Y_{2}^{(k)}\right]^{r+1}=\frac{\Gamma(r+3)}{k^{r+1}} .
\end{array}\right.
$$


Note that for $X \sim \operatorname{Exp}(1)$ we have

$$
E\left[Y_{3}^{(k)}\right]^{r}=\frac{k^{3}}{2} E X^{r+2} e^{-(k-1) X}
$$

(cf. Bieniek and Szynal [5]). Now using (1) and (2) we get

$$
E\left[Y_{2}^{(k)}\right]^{r+1}=E\left[Y_{1}^{(k)}\right]^{r+1}+\frac{(r+1) \Gamma(r+2)}{k^{r+1}}=E\left[X_{1: k}\right]^{r+1}+\frac{(r+1) \Gamma(r+2)}{k^{r+1}} .
$$

Hence we conclude that $X \sim \operatorname{Exp}(1)$ implies

$$
\left\{\begin{array}{l}
E\left[X^{r+2} e^{-(k-1) X}\right]=\frac{\Gamma(r+3)}{k^{r+3}}, \\
E\left[X_{1: k}\right]^{r+1}=\frac{\Gamma(r+2)}{k^{r+1}}
\end{array}\right.
$$

Taking into account (6) and the statement: $X \sim F$ iff $Y:=-\log (\bar{F}(X)) \sim \operatorname{Exp}(1)$ for $r>-1$ we have the implication:

$$
X \sim F \Longrightarrow\left\{\begin{array}{l}
E\left[(\bar{F}(X))^{k-1}(-\log (\bar{F}(X)))^{r+2}\right]=\frac{\Gamma(r+3)}{k^{r+3}}, \\
E\left[-\log \left(\bar{F}\left(X_{1: k}\right)\right)\right]^{r+1}=\frac{\Gamma(r+2)}{k^{r+1}}
\end{array}\right.
$$

We apply the above formulae to construct goodness-of-fit tests. The characterization condition (5) cannot be used to construct tests since in practice only a finite sample is available, whereas in general information about $Y_{n}^{(k)}$ can be obtained only from an infinite sample. The exception is $Y_{1}^{(k)}=X_{1: k}$. The method used here is to base the tests on consequences of these characterizations obtained by replacing the random variables in (5) by random variables that have the same expectations but can be estimated from any sample of size $k$ or more. Here the estimates used are $U$-statistics.

3. Tests of $H: X \sim F$ when parameters are known. We consider the hypothesis $H: X \sim F$ when $F$ is completely specified. From the sample $X_{1}, \ldots, X_{n}$ we construct tests of $H$ based on (7) for given $k \leq n$. To this end we estimate the expectations in (7) by the $U$-statistics

$$
\begin{aligned}
& \bar{V}_{n 1}^{(r, k)}=\frac{1}{n} \sum_{i=1}^{n}\left(\bar{F}\left(X_{i}\right)\right)^{k-1} \log ^{r+2} \frac{1}{\bar{F}\left(X_{i}\right)}, \\
& \bar{V}_{n 2}^{(r, k)}=\frac{1}{\left(\begin{array}{l}
n \\
k
\end{array}\right)} \sum_{i=1}^{n-k+1}\left(\begin{array}{l}
n-i \\
k-1
\end{array}\right) \log ^{r+1} \frac{1}{\bar{F}\left(X_{i: n}\right)} .
\end{aligned}
$$

We first construct tests of $H_{1}: X \sim \operatorname{Exp}(1)$, i.e. with $F(x)=1-e^{-x}, x>0$, and then we reject $H_{1}$ if these estimates differ sufficiently from the expectations in (6). Under $H_{1},(7)$ appears as (6), and the above estimates are

$$
\bar{V}_{n 1}^{(r, k)}=\frac{1}{n} \sum_{i=1}^{n} X_{i}^{r+2} e^{-(k-1) X_{i}}, \quad \bar{V}_{n 2}^{(r, k)}=\frac{1}{\left(\begin{array}{l}
n \\
k
\end{array}\right)} \sum_{i=1}^{n-k+1}\left(\begin{array}{l}
n-i \\
k-1
\end{array}\right) X_{i: n}^{r+1} .
$$


Hence for $F(x)=1-e^{-x}$ we have

$$
\begin{aligned}
& E \bar{V}_{n 1}^{(r, k)}=E\left[\frac{1}{n} \sum_{i=1}^{n} X_{i}^{r+2} e^{-(k-1) X_{i}}\right]=\frac{\Gamma(r+3)}{k^{r+3}} \\
& E \bar{V}_{n 2}^{(r, k)}=E\left[\frac{1}{\left(\begin{array}{l}
n \\
k
\end{array}\right)} \sum_{i=1}^{n-k+1}\left(\begin{array}{l}
n-i \\
k-1
\end{array}\right) X_{i: n}^{r+1}\right]=\frac{\Gamma(r+2)}{k^{r+1}} \\
& a_{n}^{(r, k)}:=\operatorname{Var}\left(\bar{V}_{n 1}^{(r, k)}\right)=\frac{1}{n}\left[\frac{\Gamma(2 r+5)}{(2 k-1)^{2 r+5}}-\frac{\Gamma^{2}(r+3)}{k^{2 r+6}}\right] \text {, } \\
& b_{n}^{(r, k)}:=\operatorname{Cov}\left(\bar{V}_{n 1}^{(r, k)}, \bar{V}_{n 2}^{(r, k)}\right)=\frac{k}{n}\left[\frac{\Gamma(2 r+5)}{(k-1)^{r+1} k^{r+3}} B_{\frac{k-1}{2 k-1}}(r+2, r+3)\right. \\
& \left.+\frac{\Gamma(2 r+4)}{(2 k-1)^{2 r+4}}-\frac{\Gamma(r+2) \Gamma(r+3)}{k^{2 r+4}}\right] \\
& c_{n}^{(r, k)}:=\operatorname{Var}\left(\bar{V}_{n 2}^{(r, k)}\right)=\frac{1}{\left(\begin{array}{l}
n \\
k
\end{array}\right)} \sum_{j=1}^{k-1}\left(\begin{array}{l}
k \\
j
\end{array}\right)\left(\begin{array}{l}
n-k \\
k-j
\end{array}\right)\left[\frac{2 \Gamma(2 r+4)}{k^{r+1}(k-j)^{r+1}} B_{\frac{k-j}{2 k-j}}(r+2, r+2)\right. \\
& \left.+j \frac{\Gamma(2 r+3)}{(2 k-j)^{2 r+3}}-\frac{\Gamma^{2}(r+2)}{k^{2 r+2}}\right]+\frac{1}{\left(\begin{array}{l}
n \\
k
\end{array}\right)} \frac{\Gamma(2 r+3)-\Gamma^{2}(r+2)}{k^{2 r+2}},
\end{aligned}
$$

where $r>-3 / 2, k>1$ and $B_{x}(\alpha, \beta)=\int_{0}^{x} t^{\alpha-1}(1-t)^{\beta-1} d t$ denotes the incomplete beta function for $0<x<1, \alpha, \beta>0$.

Simple calculations lead to the formula for $\operatorname{Var}\left(\bar{V}_{n 1}^{(r, k)}\right)$. The formula for $\operatorname{Var}\left(\bar{V}_{n 2}^{(r, k)}\right)$ was derived in Morris and Szynal [22]. To derive $\operatorname{Cov}\left(\bar{V}_{n 1}^{(r, k)}, \bar{V}_{n 2}^{(r, k)}\right)$ we use the following formula for the covariance of two $U$-statistics (Theorem 2 in Lee [13, p. 17):

Let $U_{n}^{(1)}$ and $U_{n}^{(2)}$ be two $U$-statistics, both based on a common sample $X_{1}, \ldots, X_{n}$ but having different kernels $\psi$ and $\phi$ of degrees $k_{1}$ and $k_{2}$ respectively, with $k_{1}<k_{2}$.

Define $\sigma_{c, d}^{2}$ to be the covariance between the conditional expectations of variables $\psi_{c}\left(X_{1}, \ldots, X_{n}\right)$ and $\phi_{d}\left(X_{1}, \ldots, X_{n}\right)$, and let $|S|$ denote the number of elements in $S$.

Suppose that $c \leq d$. If $S_{1}$ is in $S_{n, k_{1}}$ and $S_{2}$ in $S_{n, k_{2}}$ with $\left|S_{1} \cap S_{2}\right|=c$, where $S_{n, k}$ denotes a $k$-subset of $\{1, \ldots, n\}$, then

and

$$
\sigma_{c, d}^{2}=\operatorname{Cov}\left(\psi\left(S_{1}\right) \phi\left(S_{2}\right)\right)
$$

$$
\operatorname{Cov}\left(U_{n}^{(1)}, U_{n}^{(2)}\right)=\frac{1}{\left(\begin{array}{c}
n \\
k_{1}
\end{array}\right)} \sum_{c=1}^{k_{2}}\left(\begin{array}{c}
k_{2} \\
c
\end{array}\right)\left(\begin{array}{c}
n-k_{2} \\
k_{1}-c
\end{array}\right) \sigma_{c, c}^{2} .
$$

Let $X_{1}, \ldots, X_{n}$ be a sample from $F$, where $F(x)=1-e^{-x}$, viz. $X_{1} \in \operatorname{Exp}(1)$. Now let $k_{1}=1, k_{2}=k$ and

$$
\begin{aligned}
U_{n}^{(1)} & =\frac{1}{n} \sum_{i=1}^{n} X_{i}^{r+2} e^{-(k-1) X_{i}}, \\
U_{n}^{(2)} & =\frac{1}{\left(\begin{array}{l}
n \\
k
\end{array}\right)} \sum_{1 \leq j_{1}<\ldots<j_{k} \leq n}\left(\min \left(X_{j_{1}}, \ldots, X_{j_{k}}\right)\right)^{r+1}=\frac{1}{\left(\begin{array}{l}
n \\
k
\end{array}\right)} \sum_{i=1}^{n-k+1}\left(\begin{array}{l}
n-i \\
k-1
\end{array}\right) X_{i: n}^{r+1} .
\end{aligned}
$$

Write

$$
\psi\left(x_{1}\right)=x_{1}^{r+2} e^{-(k-1) x_{1}}, \quad \phi\left(x_{1}, \ldots, x_{k}\right)=\left(\min \left(x_{1}, \ldots, x_{k}\right)\right)^{r+1} .
$$


We suppose first that $k>1$. Letting $c=d=1$ and $x_{1}:=z$ we have

$$
\psi_{1}(z)=z^{r+2} e^{-(k-1) z}, \quad \phi_{1}(z)=E\left[\left(\min \left(X_{1}, \ldots, X_{k}\right)^{r+1} \mid X_{1}=z\right)\right],
$$

with $Z \sim \operatorname{Exp}(1)$. Then

$$
E \psi_{1}(Z)=E Z^{r+2} e^{-(k-1) Z}=\frac{\Gamma(r+3)}{k^{r+3}} .
$$

To derive $E \phi_{1}(Z)$ we write $Y_{2, k}:=\min \left(X_{2}, \ldots, X_{k}\right)$ and define

$$
U:= \begin{cases}z & \text { if } Y_{2, k}>z \\ Y_{2, k} & \text { if } Y_{2, k}<z\end{cases}
$$

then $U$ has possible values in $[0, z]$ and its distribution consists of a lump of probability at $U=z$, viz.

$$
p=P[U=z]=P\left[Y_{2, k}>z\right]=e^{-(k-1) z}
$$

and for $y \in[0, z)$ it has a density $f_{2}(y)$ equal to the density of $Y_{2, k}$. But since $Y_{2, k} \sim$ $\operatorname{Exp}(k-1)$ then $f_{2}(y)=(k-1) e^{-(k-1) y}$. Hence

$$
E U^{r+1}=z^{r+1} e^{-(k-1) z}+(k-1) \int_{0}^{z} y^{r+1} e^{-(k-1) y} d y
$$

and

$$
E \phi_{1}(Z)=E\left[Z^{r+1} e^{-(k-1) Z}+(k-1) \int_{0}^{Z} y^{r+1} e^{-(k-1) y} d y\right]=\frac{\Gamma(r+2)}{k^{r+1}}
$$

so

$$
E \psi_{1}\left(X_{1}\right) E \phi_{1}\left(X_{1}\right)=\frac{\Gamma(r+2) \Gamma(r+3)}{k^{2(r+2)}} .
$$

Also we have

$$
\begin{aligned}
E \psi_{1}(Z) \phi_{1}(Z)=E\left[Z^{r+2} e^{-(k-1) Z}\left(Z^{r+1} e^{-(k-1) Z}+(k-1) \int_{0}^{Z} y^{r+1} e^{-(k-1) y} d y\right)\right] \\
=E Z^{2 r+3} e^{-2(k-1) Z}+(k-1) E\left[Z^{r+2} e^{-(k-1) Z} \int_{0}^{Z} y^{r+1} e^{-(k-1) y} d y\right] \\
=\frac{\Gamma(2 r+4)}{(2 k-1)^{2(r+2)}}+(k-1) \int_{0}^{\infty} z^{r+2} e^{-k z} \frac{1}{(k-1)^{r+2}} \Gamma(r+2,(k-1) z) d z \\
=\frac{\Gamma(2 r+4)}{(2 k-1)^{2(r+2)}}+\frac{k-1}{r+2} \int_{0}^{\infty} z^{2 r+4} e^{-(2 k-1) z}{ }_{1} F_{1}(1 ; r+3 ;(k-1) z) d z \\
=\frac{\Gamma(2 r+4)}{(2 k-1)^{2(r+2)}}+\frac{k-1}{r+2} \frac{\Gamma(2 r+5)}{(2 k-1)^{2 r+5}}{ }_{2} F_{1}\left(1 ; 2 r+5 ; r+3, \frac{k-1}{2 k-1}\right) \\
=\frac{\Gamma(2 r+4)}{(2 k-1)^{2(r+2)}}+\frac{k-1}{r+2} \frac{\Gamma(2 r+5)}{(2 k-1)^{2 r+5}}{ }_{2} F_{1}\left(2 r+5,1 ; r+3 ; \frac{k-1}{2 k-1}\right) \\
=\frac{\Gamma(2 r+4)}{(2 k-1)^{2(r+2)}}+\frac{\Gamma(2 r+5)}{(k-1)^{r+1} k^{r+3} B_{\frac{k-1}{2 k-1}}}(r+2, r+3)
\end{aligned}
$$

where we used properties of the incomplete gamma function $\gamma(a, z)=\int_{0}^{z} t^{a-1} e^{-t} d t$ and 
the hypergeometric function

$$
{ }_{p} F_{q}\left(a_{1}, \ldots, a_{p} ; b_{1}, \ldots, b_{q} ; z\right)=\sum_{n=0}^{\infty} \frac{\left(a_{1}\right)_{n} \ldots\left(a_{p}\right)_{n}}{\left(b_{1}\right)_{n} \ldots\left(b_{q}\right)_{n}} \frac{z^{n}}{n !}
$$

from Temme 24] and Andrews et al. [1] (cf. also Appendix in Morris and Szynal [22]). Thus

$$
E\left[\psi_{1}\left(X_{1}\right) \phi_{1}\left(X_{1}\right)\right]=\frac{\Gamma(2 r+4)}{(2 k-1)^{2(r+2)}}+\frac{\Gamma(2 r+5)}{(k-1)^{r+1} k^{r+3}} B_{\frac{k-1}{2 k-1}}(r+2, r+3) .
$$

Therefore

$$
\begin{aligned}
& \operatorname{Cov}\left(\psi_{1}\left(X_{1}\right) \phi_{1}\left(X_{1}\right)\right) \\
& =\frac{\Gamma(2 r+4)}{(2 k-1)^{2(r+2)}}+\frac{\Gamma(2 r+5)}{(k-1)^{r+1} k^{r+3}} B_{\frac{k-1}{2 k-1}}(r+2, r+3)-\frac{\Gamma(r+2)}{k^{r+1}} \frac{\Gamma(r+3)}{k^{r+3}}
\end{aligned}
$$

and

$$
\begin{aligned}
& \operatorname{Cov}\left(\bar{V}_{n 1}^{(r, k)}, \bar{V}_{n 2}^{(r, k)}\right)=\frac{k}{n} \operatorname{Cov}\left(\psi_{1}\left(X_{1}\right) \phi_{1}\left(X_{1}\right)\right) \\
& =\frac{k}{n}\left[\frac{\Gamma(2 r+4)}{(2 k-1)^{2(r+2)}}-\frac{\Gamma(r+2) \Gamma(r+3)}{k^{2(r+2)}}+\frac{\Gamma(2 r+5)}{(k-1)^{r+1} k^{r+3}} B_{\frac{k-1}{2 k-1}}(r+1, r+3)\right] .
\end{aligned}
$$

When $k=1$ simple calculations (after letting $B_{\frac{k-1}{2 k-1}}(r+1, r+3) /(k-1)^{r+1}=0$ ) show that

$$
\Sigma^{(r, 1)}=\left[\begin{array}{cc}
\left(\sigma_{1}^{(r, 1)}\right)^{2} & \sigma_{12}^{(r, 1)} \\
\sigma_{12}^{(r, 1)} & \left(\sigma_{2}^{(r, 1)}\right)^{2}
\end{array}\right]
$$

where

$$
\begin{aligned}
\left(\sigma_{1}^{(r, 1)}\right)^{2} & =\Gamma(2 r+5)-\Gamma^{2}(r+3), \quad\left(\sigma_{2}^{(r, 1)}\right)^{2}=\Gamma(2 r+3)-\Gamma^{2}(r+2) \\
\sigma_{12}^{(r, 1)} & =\Gamma(2 r+4)-\Gamma(r+2) \Gamma(r+3) .
\end{aligned}
$$

Note that $\left(\overline{\boldsymbol{V}}_{n}^{(r, k)}-\boldsymbol{\mu}_{0}\right)$ is asymptotically Normal. Hence tests of $H_{1}: X \sim \operatorname{Exp}(1)$ based on the implication (6) can be obtained from

$$
T_{n}^{(r, k)}=\left(\overline{\boldsymbol{V}}_{n}^{(r, k)}-\boldsymbol{\mu}_{0}\right)^{\prime}\left(\Sigma_{n}^{(r, k)}\right)^{-1}\left(\overline{\boldsymbol{V}}_{n}^{(r, k)}-\boldsymbol{\mu}_{0}\right) \stackrel{D}{\rightarrow} \chi^{2}(2) .
$$

with

$$
\boldsymbol{\mu}_{0}=\frac{\Gamma(r+2)}{k^{r+1}}\left[\begin{array}{c}
\frac{r+2}{k^{2}} \\
1
\end{array}\right], \quad \Sigma_{n}^{(r, k)}=\left[\begin{array}{ll}
a_{n}^{(r, k)} & b_{n}^{(r, k)} \\
b_{n}^{(r, k)} & c_{n}^{(r, k)}
\end{array}\right] .
$$

If $X$ has a continuous distribution with specified distribution function $F$ then tests of $H$ are obtained from $T_{n}^{(r, k)}$ in $(8)$ by replacing the sample $X_{1}, \ldots, X_{n}$ by $Y_{1}, \ldots, Y_{n}$ with $Y=h(X)=\log \frac{1}{\bar{F}(X)}$.

Since $h$ is an increasing function then, referring to the definition of $\bar{V}_{n 2}^{(r, k)}$ (at the beginning of Section 3), in this case $Y_{1: k}^{\prime}=\min \left(Y_{i_{1}}, Y_{i_{2}}, \ldots, Y_{i_{k}}\right)=h\left(X_{1: k}^{\prime}\right)$, where $X_{i: k}^{\prime}=$ $\min \left(X_{i_{1}}, \ldots, X_{i_{k}}\right), 1 \leq i_{1}<i_{2}<\ldots<i_{k} \leq n$, so that

$$
\bar{V}_{n 2}^{(r, k)}=\frac{1}{\left(\begin{array}{l}
n \\
k
\end{array}\right)} \sum^{\prime} \log ^{r+1} \frac{1}{\bar{F}\left(X_{1: k}^{\prime}\right)}=\frac{1}{\left(\begin{array}{l}
n \\
k
\end{array}\right)} \sum_{i=1}^{n-k+1}\left(\begin{array}{l}
n-i \\
k-1
\end{array}\right) \log ^{r+1} \frac{1}{\bar{F}\left(X_{i: k}\right)}
$$


where $\sum^{\prime}$ denotes summation over all $1 \leq i_{1}<i_{2}<\ldots<i_{k} \leq n$. The test statistic in (8) is thus

$$
T_{n}^{(r, k)}=\left(\overline{\boldsymbol{V}}_{n}^{(r, k)}-\boldsymbol{\mu}_{0}\right)^{\prime}\left(\Sigma_{n}^{(r, k)}\right)^{-1}\left(\overline{\boldsymbol{V}}_{n}^{(r, k)}-\boldsymbol{\mu}_{0}\right) \stackrel{D}{\rightarrow} \chi^{2}(2)
$$

where

$$
\overline{\boldsymbol{V}}_{n}^{(r, k)}=\left[\begin{array}{l}
\bar{V}_{n 1}^{(r, k)} \\
\bar{V}_{n 2}^{(r, k)}
\end{array}\right]
$$

with

$$
\bar{V}_{n 1}^{(r, k)}=\frac{1}{n} \sum_{i=1}^{n}\left(\bar{F}\left(X_{i}\right)\right)^{k-1} \log ^{r+2} \frac{1}{\bar{F}\left(X_{i}\right)}, \quad \bar{V}_{n 2}^{(r, k)}=\frac{1}{\left(\begin{array}{l}
n \\
k
\end{array}\right)} \sum_{i=1}^{n-k+1}\left(\begin{array}{l}
n-i \\
k-1
\end{array}\right) \log ^{r+1} \frac{1}{\bar{F}\left(X_{i: n}\right)} .
$$

Taking into account that

$$
\left(\Sigma_{n}^{(r, k)}\right)^{-1}=\frac{1}{\Delta_{n}^{(r, k)}}\left[\begin{array}{cc}
c_{n}^{(r, k)} & -b_{n}^{(r, k)} \\
-b_{n}^{(r, k)} & a_{n}^{(r, k)}
\end{array}\right], \quad \text { where } \quad \Delta_{n}^{(r, k)}=\operatorname{det}\left(\Sigma_{n}^{(r, k)}\right),
$$

we can write $T_{n}^{(r, k)}$ in the following extended form

$$
\begin{aligned}
& T_{n}^{(r, k)}=\frac{1}{\Delta_{n}^{(r, k)}}\left[c_{n}^{(r, k)}\left(\bar{V}_{n 1}^{(r, k)}-\frac{\Gamma(r+3)}{k^{r+3}}\right)^{2}\right. \\
& \left.-2 b_{n}^{(r, k)}\left(\bar{V}_{n 1}^{(r, k)}-\frac{\Gamma(r+3)}{k^{r+3}}\right)\left(\bar{V}_{n 2}^{(r, k)}-\frac{\Gamma(r+2)}{k^{r+1}}\right)+a_{n}^{(r, k)}\left(\bar{V}_{n 2}^{(r, k)}-\frac{\Gamma(r+2)}{k^{r+1}}\right)^{2}\right] .
\end{aligned}
$$

We also consider tests using the statistics obtained from the following partitions:

$$
T_{n}^{(r, k)}=T_{n ; c_{1}}^{(r, k)}+T_{n ; c_{2}}^{(r, k)}=T_{n ; c_{3}}^{(r, k)}+T_{n ; c_{4}}^{(r, k)}
$$

where

$$
\begin{aligned}
& T_{n ; c_{1}}^{(r, k)}=\frac{1}{a_{n}^{(r, k)}}\left(\bar{V}_{n 1}^{(r, k)}-\frac{\Gamma(r+3)}{k^{r+3}}\right)^{2}, \\
& T_{n ; c_{2}}^{(r, k)}=\frac{1}{\Delta_{n}^{(r, k)} a_{n}^{(r, k)}}\left[a_{n}^{(r, k)} \bar{V}_{n 2}^{(r, k)}-b_{n}^{(r, k)} \bar{V}_{n 1}^{(r, k)}-\frac{\Gamma(r+2)}{k^{r+3}}\left(k^{2} a_{n}^{(r, k)}-(r+2) b_{n}^{(r, k)}\right)\right]^{2}, \\
& T_{n ; c_{3}}^{(r, k)}=\frac{1}{c_{n}^{(r, k)}}\left(\bar{V}_{n 2}^{(r, k)}-\frac{\Gamma(r+2)}{k^{r+1}}\right)^{2}, \\
& T_{n ; c_{4}}^{(r, k)}=\frac{1}{\Delta_{n}^{(r, k)} c_{n}^{(r, k)}}\left[c_{n}^{(r, k)} \bar{V}_{n 1}^{(r, k)}-b_{n}^{(r, k)} \bar{V}_{n 2}^{(r, k)}-\frac{\Gamma(r+2)}{k^{r+3}}\left((r+2) c_{n}^{(r, k)}-k^{2} b_{n}^{(r, k)}\right)\right]^{2} .
\end{aligned}
$$

4. Tests of $H: X \sim F$ when there are unknown parameters. We now study the hypothesis $H$ that $F$ has the form $F(x ; \boldsymbol{\lambda})$ where $\boldsymbol{\lambda}(p \times 1)$ are identifiable parameters with unknown true value $\boldsymbol{\lambda}_{0}$ in the parameter space $\Lambda$, and we denote the pdf by $f(x ; \boldsymbol{\lambda})$.

Here we define

$$
\hat{\boldsymbol{V}}_{n}^{(r, k)}=\left[\begin{array}{c}
\hat{V}_{n 1}^{(r, k)} \\
\hat{V}_{n 2}^{(r, k)}
\end{array}\right]
$$


where

$$
\begin{aligned}
\hat{V}_{n 1}^{(r, k)}: & =\bar{V}_{n 1}^{(r, k)}\left(\hat{\boldsymbol{\lambda}}_{n}\right)=\frac{1}{n} \sum_{i=1}^{n}\left(\bar{F}\left(X_{i} ; \hat{\boldsymbol{\lambda}}_{n}\right)\right)^{k-1} \log { }^{r+2} \frac{1}{\bar{F}\left(X_{i} ; \hat{\boldsymbol{\lambda}}_{n}\right)}, \\
\hat{V}_{n 2}^{(r, k)}: & =\bar{V}_{n 2}^{(r, k)}\left(\hat{\boldsymbol{\lambda}}_{n}\right)=\frac{1}{\left(\begin{array}{l}
n \\
k
\end{array}\right)} \sum_{i=1}^{n-k+1}\left(\begin{array}{c}
n-i \\
k-1
\end{array}\right) \log ^{r+1} \frac{1}{\bar{F}\left(X_{i: n} ; \hat{\boldsymbol{\lambda}}_{n}\right)},
\end{aligned}
$$

where $\hat{\boldsymbol{\lambda}}_{n}$ is the MLE of $\boldsymbol{\lambda}$. We then construct tests of $H$ as in Section 3, but using $F\left(x ; \hat{\lambda}_{n}\right)$ instead of $F(x)$. And we further assume regular estimation, for which

$$
\sqrt{n}\left(\hat{\boldsymbol{\lambda}}_{n}-\boldsymbol{\lambda}_{0}\right) \stackrel{D}{\rightarrow} N\left(\mathbf{0}, I^{-1}\left(\boldsymbol{\lambda}_{0}\right)\right)
$$

where $I\left(\boldsymbol{\lambda}_{0}\right)$ is the expected information matrix based on a single observation $X$ from $F\left(x ; \lambda_{0}\right)$.

Following the procedure presented for this case where a theorem of Pierce [23] is used (cf. Morris and Szynal [17]) for a sample from $F\left(x ; \boldsymbol{\lambda}_{0}\right)$ the variance matrix of $\hat{\boldsymbol{V}}_{n}^{(r, k)}$ for large $n$ is given approximately by

$$
\Sigma_{n 1}^{(r, k)}\left(\boldsymbol{\lambda}_{0}\right)=\Sigma_{n}^{(r, k)}-\frac{1}{n} \boldsymbol{B}_{n}^{(r, k)}\left(\boldsymbol{\lambda}_{0}\right) \mathcal{I}^{-1}\left(\boldsymbol{\lambda}_{0}\right)\left(\boldsymbol{B}_{n}^{(r, k)}\left(\boldsymbol{\lambda}_{0}\right)\right)^{\prime}=\Sigma_{n}^{(r, k)}-\boldsymbol{K}_{n}^{(r, k)}\left(\boldsymbol{\lambda}_{0}\right)
$$

with

$$
\boldsymbol{B}_{n}^{(r, k)}(2 \times p)=E_{0}\left[\frac{\partial \overline{\boldsymbol{V}}_{n}^{(r, k)}(\boldsymbol{\lambda})}{\partial \boldsymbol{\lambda}}\right]
$$

where $E_{0}$ means expectation when $X$ is $F\left(x ; \lambda_{0}\right)$, and

$$
\boldsymbol{K}_{n}^{(r, k)}=\frac{1}{n} \boldsymbol{B}_{n}^{(r, k)} \mathcal{I}^{-1}\left(\boldsymbol{B}_{n}^{(r, k)}\right)^{\prime} .
$$

Here

$$
\begin{aligned}
& b_{1}^{(r, k)}\left(\lambda_{j}\right):=E \frac{\partial \hat{V}_{n 1}^{(r, k)}(\boldsymbol{\lambda})}{\partial \lambda_{j}}=\frac{1}{n} \sum_{i=1}^{n} E\left[\frac{\partial}{\partial \lambda_{j}}\left(\bar{F}\left(X_{i} ; \boldsymbol{\lambda}\right)\right)^{k-1} \log ^{r+2} \frac{1}{\bar{F}\left(X_{i} ; \boldsymbol{\lambda}\right)}\right] \\
& =E\left[\frac{\partial}{\partial \lambda_{j}}(\bar{F}(X ; \boldsymbol{\lambda}))^{k-1} \log ^{r+2} \overline{\bar{F}(X ; \boldsymbol{\lambda})}\right] \\
& \quad=E\left\{[r+2+(k-1) \log (\bar{F}(X ; \boldsymbol{\lambda}))](\bar{F}(X ; \boldsymbol{\lambda}))^{k-2} \log ^{r+1} \frac{1}{\bar{F}(X ; \boldsymbol{\lambda})} \frac{\partial F(X ; \boldsymbol{\lambda})}{\partial \lambda_{j}}\right\},
\end{aligned}
$$

and

$$
b_{2}^{(r, k)}\left(\lambda_{j}\right):=E \frac{\partial \hat{V}_{n 2}^{(r, k)}(\boldsymbol{\lambda})}{\partial \lambda_{j}}=k(r+1) E\left[(\bar{F}(X ; \boldsymbol{\lambda}))^{k-2} \log ^{r} \frac{1}{\bar{F}(X ; \boldsymbol{\lambda})} \frac{\partial F(X ; \boldsymbol{\lambda})}{\partial \lambda_{j}}\right]
$$

(cf. Morris and Szynal [22]). Write

$$
b^{(r, k)}\left(\lambda_{j}\right)=E\left[(\bar{F}(X ; \boldsymbol{\lambda}))^{k-2} \log ^{r} \frac{1}{\bar{F}(X ; \boldsymbol{\lambda})} \frac{\partial F(X ; \boldsymbol{\lambda})}{\partial \lambda_{j}}\right],
$$

$j=1, \ldots, p$. Then 


$$
\begin{aligned}
& \boldsymbol{B}_{n}^{(r, k)}:=\boldsymbol{B}^{(r, k)}=\left[\begin{array}{ccc}
b_{1}^{(r, k)}\left(\lambda_{1}\right) & \ldots & b_{1}^{(r, k)}\left(\lambda_{p}\right) \\
b_{2}^{(r, k)}\left(\lambda_{1}\right) & \ldots & b_{2}^{(r, k)}\left(\lambda_{p}\right)
\end{array}\right] \\
& =\left[\begin{array}{ccc}
(r+2) b^{(r+1, k)}\left(\lambda_{1}\right)-(k-1) b^{(r+2, k)}\left(\lambda_{1}\right) & \ldots & (r+2) b^{(r+1, k)}\left(\lambda_{p}\right)-(k-1) b^{(r+2, k)}\left(\lambda_{p}\right) \\
k(r+1) b^{(r, k)}\left(\lambda_{1}\right) & \ldots & k(r+1) b^{(r, k)}\left(\lambda_{p}\right)
\end{array}\right]
\end{aligned}
$$

and

$$
\boldsymbol{K}_{n}^{(r, k)}=\frac{1}{n} \boldsymbol{B}_{n}^{(r, k)} \mathcal{I}^{-1}\left(\boldsymbol{B}_{n}^{(r, k)}\right)^{\prime}:=\left[\begin{array}{ll}
s_{n}^{(r, k)} & t_{n}^{(r, k)} \\
t_{n}^{(r, k)} & u_{n}^{(r, k)}
\end{array}\right] .
$$

Thus corresponding to $T_{n}^{(r, k)}$ in $(8)$ we have

$$
\hat{T}_{n}^{(r, k)}=\hat{T}_{n}^{(r, k)}\left(\boldsymbol{\lambda}_{0}\right)=\left(\hat{\boldsymbol{V}}_{n}^{(r, k)}-\boldsymbol{\mu}_{0}\right)^{\prime}\left(\Sigma_{n 1}^{(r, k)}\left(\boldsymbol{\lambda}_{0}\right)\right)^{-1}\left(\hat{\boldsymbol{V}}_{n}^{(r, k)}-\boldsymbol{\mu}_{0}\right) \stackrel{D}{\rightarrow} \chi^{2}(2)
$$

under $H$, but this can be used for testing $H$ only if it does not depend on the unknown $\boldsymbol{\lambda}_{0}$, i.e. if $\boldsymbol{K}^{(r, k)}\left(\boldsymbol{\lambda}_{0}\right)$ does not depend on $\boldsymbol{\lambda}_{0}$.

In one or two parameters when $\boldsymbol{\lambda}=(\alpha)$ and $\boldsymbol{\lambda}=(\alpha, \beta)$ we write

$$
I^{-1}:=\left[i_{11}\right] \quad \text { and } \quad I^{-1}:=\left[\begin{array}{ll}
i_{11} & i_{12} \\
i_{12} & i_{22}
\end{array}\right]
$$

and then for $\boldsymbol{\lambda}=(\alpha)$ we have

$$
\begin{aligned}
s_{n}^{(r, k)} & =\frac{1}{n}\left(b_{1}^{(r, k)}(\alpha)\right)^{2} i_{11}=\frac{1}{n}\left[(r+2) b^{(r+1, k)}(\alpha)-(k-1) b^{(r+2, k)}(\alpha)\right]^{2} i_{11}, \\
t_{n}^{(r, k)} & =\frac{1}{n} b_{1}^{(r, k)}(\alpha) b_{2}^{(r, k)}(\alpha) i_{11} \\
& =\frac{k(r+1)}{n}\left[(r+2) b^{(r+1, k)}(\alpha)-(k-1) b^{(r+2, k)}(\alpha)\right] b^{(r, k)}(\alpha) i_{11}, \\
u_{n}^{(r, k)} & =\frac{1}{n}\left(b_{2}^{(r, k)}(\alpha)\right)^{2} i_{11}=\frac{k^{2}(r+1)^{2}}{n}\left(b^{(r, k)}(\alpha)\right)^{2} i_{11},
\end{aligned}
$$

with

$$
b^{(r, k)}(\alpha)=E\left[(\bar{F}(X ; \alpha))^{k-2} \log ^{r} \frac{1}{\bar{F}(X ; \alpha)} \frac{\partial F(X ; \alpha)}{\partial \alpha}\right]
$$

and for $\boldsymbol{\lambda}=(\alpha, \beta)$ we have

$$
\begin{aligned}
s_{n}^{(r, k)}= & \frac{1}{n}\left[\left(b_{1}^{(r, k)}(\alpha)\right)^{2} i_{11}+2 b_{1}^{(r, k)}(\alpha) b_{1}^{(r, k)}(\beta) i_{12}+\left(b_{1}^{(r, k)}(\beta)\right)^{2} i_{22}\right] \\
= & \frac{1}{n}\left\{\left[(r+2) b^{(r+1, k)}(\alpha)-(k-1) b^{(r+2, k)}(\alpha)\right]^{2} i_{11}\right. \\
& +2\left[(r+2) b^{(r+1, k)}(\alpha)-(k-1) b^{(r+2, k)}(\alpha)\right] \\
& \cdot\left[(r+2) b^{(r+1, k)}(\beta)-(k-1) b^{(r+2, k)}(\beta)\right] i_{12} \\
& \left.+\left[(r+2) b^{(r+1, k)}(\beta)-(k-1) b^{(r+2, k)}(\beta)\right]^{2} i_{22}\right\}, \\
t_{n}^{(r, k)}= & \frac{1}{n}\left[b_{1}^{(r, k)}(\alpha) b_{2}^{(r+1, k)}(\alpha) i_{11}+\left(b_{1}^{(r, k)}(\alpha) b_{2}^{(r+1, k)}(\beta)+b_{1}^{(r+1, k)}(\alpha) b_{2}^{(r, k)}(\beta)\right) i_{12}\right. \\
& \left.+b_{1}^{(r, k)}(\beta) b_{2}^{(r+1, k)}(\beta) i_{22}\right]
\end{aligned}
$$




$$
\begin{aligned}
= & \frac{k(r+1)}{n}\left\{\left[(r+2) b^{(r+1, k)}(\alpha)-(k-1) b^{(r+2, k)}(\alpha)\right] b^{(r, k)}(\alpha) i_{11}\right. \\
& +\left(\left[(r+2) b^{(r+1, k)}(\alpha)-(k-1) b^{(r+2, k)}(\beta)\right] b^{(r, k)}(\alpha)\right. \\
& \left.+\left[(r+2) b^{(r+1, k)}(\alpha)-(k-1) b^{(r+2, k)}(\alpha)\right] b^{(r, k)}(\beta)\right) i_{12} \\
& \left.+\left[(r+2) b^{(r+1, k)}(\beta)-(k-1) b^{(r+2, k)}(\beta)\right] b^{(r, k)} i_{22}\right\}, \\
u_{n}^{(r, k)}= & \frac{k^{2}(r+1)^{2}}{n}\left[\left(b^{(r, k)}(\alpha)\right)^{2} i_{11}+2 b^{(r, k)}(\alpha) b^{(r, k)}(\beta) i_{12}+\left(b^{(r, k)}(\beta)\right)^{2} i_{22}\right]
\end{aligned}
$$

with

$$
\begin{aligned}
& b^{(r, k)}(\alpha)=E\left[(\bar{F}(X ; \alpha, \beta))^{k-2} \log ^{r} \frac{1}{\bar{F}(X ; \alpha, \beta)} \frac{\partial F(X ; \alpha, \beta)}{\partial \alpha}\right], \\
& b^{(r, k)}(\beta)=E\left[(\bar{F}(X ; \alpha, \beta))^{k-2} \log ^{r} \overline{\bar{F}(X ; \alpha, \beta)} \frac{1}{\partial \beta}\right]
\end{aligned}
$$

Write

$$
\left(\Sigma_{n 1}^{(r, k)}\right)^{-1}=\frac{1}{\Delta_{n 1}^{(r, k)}}\left[\begin{array}{cc}
c_{n 1}^{(r, k)} & -b_{n 1}^{(r, k)} \\
-b_{n 1}^{(r, k)} & a_{n 1}^{(r, k)}
\end{array}\right], \quad \Delta_{n 1}^{(r, k)}=\operatorname{det}\left(\Sigma_{n 1}^{(r, k)}\right)
$$

Then in extended form the above test statistic $\hat{T}_{n}^{(r, k)}$ is

$$
\begin{aligned}
\hat{T}_{n}^{(r, k)}= & \frac{1}{\Delta_{n 1}^{(r, k)}}\left[c_{n 1}^{(r, k)}\left(\hat{V}_{n 1}^{(r, k)}-\frac{\Gamma(r+3)}{k^{r+3}}\right)^{2}-2 b_{n 1}^{(r, k)}\left(\hat{V}_{n 1}^{(r, k)}-\frac{\Gamma(r+3)}{k^{r+3}}\right)\left(\hat{V}_{n 2}^{(r, k)}-\frac{\Gamma(r+2)}{k^{r+1}}\right)\right. \\
& \left.+a_{n 1}^{(r, k)}\left(\hat{V}_{n 2}^{(r, k)}-\frac{\Gamma(r+2)}{k^{r+1}}\right)^{2}\right]
\end{aligned}
$$

and we use the partitions

$$
\hat{T}_{n}^{(r, k)}=\hat{T}_{n ; c_{1}}^{(r, k)}+\hat{T}_{n ; c_{2}}^{(r, k)}=\hat{T}_{n ; c_{3}}^{(r, k)}+\hat{T}_{n ; c_{4}}^{(r, k)}
$$

where

$$
\begin{aligned}
& \hat{T}_{n ; c_{1}}^{(r, k)}=\frac{1}{a_{n 1}^{(r, k)}}\left(\hat{V}_{n 1}^{(r, k)}-\frac{\Gamma(r+3)}{k^{r+3}}\right)^{2}, \\
& \hat{T}_{n ; c_{2}}^{(r, k)}=\frac{1}{\Delta_{n 1}^{(r, k)} a_{n 1}^{(r, k)}}\left[a_{n 1}^{(r, k)} \hat{V}_{n 2}^{(r, k)}-b_{n 1}^{(r, k)} \hat{V}_{n 1}^{(r, k)}-\frac{\Gamma(r+2)}{k^{r+3}}\left(k^{2} a_{n 1}^{(r, k)}-(r+2) b_{n 1}^{(r, k)}\right)\right]^{2}, \\
& \hat{T}_{n ; c_{3}}^{(r, k)}=\frac{1}{c_{n 1}^{(r, k)}}\left(\hat{V}_{n 2}^{(r, k)}-\frac{\Gamma(r+2)}{k^{r+1}}\right)^{2}, \\
& \hat{T}_{n ; c_{4}}^{(r, k)}=\frac{1}{\Delta_{n 1}^{(r, k)} c_{n 1}^{(r, k)}}\left[c_{n 1}^{(r, k)} \hat{V}_{n 1}^{(r, k)}-b_{n 1}^{(r, k)} \hat{V}_{n 2}^{(r, k)}-\frac{\Gamma(r+2)}{k^{r+3}}\left((r+2) c_{n 1}^{(r, k)}-k^{2} b_{n 1}^{(r, k)}\right)\right]^{2} .
\end{aligned}
$$




\section{Special cases}

5.1. Exponential distribution: $X \sim \boldsymbol{E} \operatorname{xp}(\alpha)$. Here $F(x ; \alpha)=1-e^{-\alpha x}$ for $x>0$, $\Lambda=\{\alpha: \alpha>0\}, I^{-1}=\alpha^{2}$ and

$$
\begin{aligned}
& \hat{\alpha}_{n}=\frac{1}{\bar{X}_{n}}, \quad \bar{X}_{n}=\frac{1}{n} \sum_{i=1}^{n} X_{i}, \\
& b^{(r, k)}(\alpha)=E\left[(\bar{F}(X ; \alpha))^{k-2} \log ^{r} \frac{1}{\bar{F}(X ; \alpha)} \frac{\partial F(X ; \alpha)}{\partial \alpha}\right]=\frac{1}{\alpha} \frac{\Gamma(r+2)}{k^{r+2}} .
\end{aligned}
$$

Then we can write $s_{n}^{(r, k)}, t_{n}^{(r, k)}$ and $u_{n}^{(r, k)}$ as follows

$$
\begin{aligned}
s_{n}^{(r, k)} & =\frac{1}{n}\left(\frac{\Gamma(r+4)-k \Gamma(r+3)}{k^{r+4}}\right)^{2}, \\
t_{n}^{(r, k)} & =\frac{1}{n} \frac{(\Gamma(r+3)-\Gamma(r+2))(\Gamma(r+4)-k \Gamma(r+3))}{k^{2 r+5}}, \\
u_{n}^{(r, k)} & =\frac{1}{n}\left(\frac{\Gamma(r+3)-\Gamma(r+2)}{k^{r+1}}\right)^{2} .
\end{aligned}
$$

Referring to $\Sigma_{n 1}^{(r, k)}$ it follows that for $k>1$

$$
\begin{aligned}
& a_{n 1}^{(r, k)}=a_{n}^{(r, k)}-\frac{1}{n}\left(\frac{\Gamma^{2}(r+4)-k \Gamma(r+3)}{k^{r+4}}\right)^{2}, \\
& =\frac{1}{n}\left[\frac{\Gamma(2 r+5)}{(2 k-1)^{2 r+5}}-\frac{\Gamma^{2}(r+4)}{k^{2 r+8}}+\frac{2 \Gamma(r+3)(\Gamma(r+4)-k \Gamma(r+3))}{k^{2 r+7}}\right] \text {, } \\
& b_{n 1}^{(r, k)}=b_{n}^{(r, k)}-\frac{1}{n} \frac{(\Gamma(r+3)-\Gamma(r+2))(\Gamma(r+4)-k \Gamma(r+3))}{k^{2 r+5}} \\
& =\frac{k}{n}\left[\frac{\Gamma(2 r+5)}{(k-1)^{r+1} k^{r+3}} B_{\frac{k-1}{2 k-1}}(r+2, r+3)+\frac{\Gamma(2 r+4)}{(2 k-1)^{2 r+4}}\right. \\
& \left.-\frac{\Gamma(r+2) \Gamma(r+3)}{k^{2 r+4}}-\frac{(\Gamma(r+3)-\Gamma(r+2))(\Gamma(r+4)-k \Gamma(r+3))}{k^{2 r+6}}\right], \\
& c_{n 1}^{(r, k)}=c_{n}^{(r, k)}-\frac{(\Gamma(r+3)-\Gamma(r+2))^{2}}{n k^{2 r+2}} \\
& =\frac{1}{\left(\begin{array}{l}
n \\
k
\end{array}\right)} \sum_{j=1}^{k-1}\left(\begin{array}{l}
k \\
j
\end{array}\right)\left(\begin{array}{l}
n-k \\
k-j
\end{array}\right)\left[2 \frac{\Gamma(2 r+4)}{k^{r+1}(k-j)^{r+1}} B_{\frac{k-j}{2 k-j}}(r+2, r+2)\right. \\
& \left.+j \frac{\Gamma(2 r+3)}{(2 k-j)^{2 r+3}}-\frac{\Gamma^{2}(r+2)}{k^{2 r+2}}\right]+\frac{1}{\left(\begin{array}{l}
n \\
k
\end{array}\right)}\left[\frac{\Gamma(2 r+3)-\Gamma^{2}(r+2)}{k^{2 r+2}}\right] \\
& -\frac{(\Gamma(r+3)-\Gamma(r+2))^{2}}{n k^{2 r+2}}
\end{aligned}
$$

and when $k=1$

$$
\begin{aligned}
& a_{n 1}^{(r, 1)}=\frac{1}{n}\left[\Gamma(2 r+5)-\Gamma^{2}(r+4)+2 \Gamma(r+3)(\Gamma(r+4)-\Gamma(r+3))\right], \\
& b_{n 1}^{(r, 1)}=\frac{1}{n}[\Gamma(2 r+4)-\Gamma(r+2) \Gamma(r+3)-(\Gamma(r+3)-\Gamma(r+2))(\Gamma(r+4)-\Gamma(r+3))],
\end{aligned}
$$




$$
c_{n 1}^{(r, 1)}=\frac{1}{n}\left[\Gamma(2 r+3)-\Gamma^{2}(r+2)-(\Gamma(r+3)-\Gamma(r+2))^{2}\right] .
$$

For the direct tests we have

$$
\begin{aligned}
\hat{T}_{n}^{(r, k)}= & \frac{1}{\Delta_{n 1}^{(r, k)}}\left[c_{n 1}^{(r, k)}\left(\frac{1}{n} \sum_{i=1}^{n}\left(\frac{X_{i}}{\bar{X}_{n}}\right)^{r+2} e^{-(k-1) X_{i} / \bar{X}_{n}}-\frac{\Gamma(r+3)}{k^{r+3}}\right)^{2}\right. \\
& -2 b_{n 1}^{(r, k)}\left(\frac{1}{n} \sum_{i=1}^{n}\left(\frac{X_{i}}{\bar{X}_{n}}\right)^{r+2} e^{-(k-1) X_{i} / \bar{X}_{n}}-\frac{\Gamma(r+3)}{k^{r+3}}\right) \\
& \cdot\left(\frac{1}{\left(\begin{array}{l}
n \\
k
\end{array}\right)} \sum_{i=1}^{n-k+1}\left(\begin{array}{l}
n-i \\
k-1
\end{array}\right)\left(\frac{X_{i: n}}{\bar{X}_{n}}\right)^{r+1}-\frac{\Gamma(r+2)}{k^{r+1}}\right) \\
& \left.+a_{n 1}^{(r, k)}\left(\frac{1}{\left(\begin{array}{l}
n \\
k
\end{array}\right)} \sum_{i=1}^{n-k+1}\left(\begin{array}{l}
n-i \\
k-1
\end{array}\right)\left(\frac{X_{i: n}}{\bar{X}_{n}}\right)^{r+1}-\frac{\Gamma(r+2)}{k^{r+1}}\right)^{2}\right] \\
= & \hat{T}_{n ; c_{1}}^{(r, k)}+\hat{T}_{n ; c_{2}}^{(r, k)}=\hat{T}_{n ; c_{3}}^{(r, k)}+\hat{T}_{n ; c_{4}}^{(r, k)}
\end{aligned}
$$

where

$$
\begin{aligned}
& \hat{T}_{n ; c_{1}}^{(r, k)}=\frac{1}{a_{n 1}^{(r, k)}}\left[\frac{1}{n} \sum_{i=1}^{n}\left(\frac{X_{i}}{\bar{X}_{n}}\right)^{r+2} e^{-(k-1) X_{i} / \bar{X}_{n}}-\frac{\Gamma(r+3)}{k^{r+3}}\right]^{2}, \\
& \hat{T}_{n ; c_{2}}^{(r, k)}=\frac{1}{\Delta_{n 1}^{(r, k)} a_{n 1}^{(r, k)}}\left[a_{n 1}^{(r, k)} \frac{1}{\left(\begin{array}{l}
n \\
k
\end{array}\right)} \sum_{i=1}^{n-k+1}\left(\begin{array}{l}
n-i \\
k-1
\end{array}\right)\left(\frac{X_{i: n}}{\bar{X}_{n}}\right)^{r+1}\right. \\
& \left.-b_{n 1}^{(r, k)} \frac{1}{n} \sum_{i=1}^{n}\left(\frac{X_{i}}{\bar{X}_{n}}\right)^{r+2} e^{-(k-1) X_{i} / \bar{X}_{n}}-\frac{\Gamma(r+2)}{k^{r+3}}\left(k^{2} a_{n 1}^{(r, k)}-(r+2) b_{n 1}^{(r, k)}\right)\right]^{2}, \\
& \hat{T}_{n ; c_{3}}^{(r, k)}=\frac{1}{c_{n 1}^{(r, k)}}\left[\frac{1}{\left(\begin{array}{l}
n \\
k
\end{array}\right)} \sum_{i=1}^{n-k+1}\left(\begin{array}{l}
n-i \\
k-1
\end{array}\right)\left(\frac{X_{i: n}}{\bar{X}_{n}}\right)^{r+1}-\frac{\Gamma(r+2)}{k^{r+1}}\right]^{2}, \\
& \hat{T}_{n ; c_{4}}^{(r, k)}=\frac{1}{\Delta_{n 1}^{(r, k)} c_{n 1}^{(r, k)}}\left[c_{n 1}^{(r, k)} \frac{1}{n} \sum_{i=1}^{n}\left(\frac{X_{i}}{\bar{X}_{n}}\right)^{r+2} e^{-(k-1) X_{i} / \bar{X}_{n}}\right. \\
& \left.-b_{n 1}^{(r, k)} \frac{1}{\left(\begin{array}{l}
n \\
k
\end{array}\right)} \sum_{i=1}^{n-k+1}\left(\begin{array}{c}
n-i \\
k-1
\end{array}\right)\left(\frac{X_{i: n}}{\bar{X}_{n}}\right)^{r+1}-\frac{\Gamma(r+2)}{k^{r+3}}\left((r+2) c_{n 1}^{(r, k)}-k^{2} b_{n 1}^{(r, k)}\right)\right]^{2} \text {. }
\end{aligned}
$$

5.2. Rayleigh distribution: $X \sim \operatorname{Ral}(\alpha)$. Here $F(x):=F(x ; \alpha)=1-e^{-\alpha x^{2}}, f(x)=$ $2 \alpha x e^{-\alpha x^{2}}$ for $x>0, \Lambda=\{\alpha: \alpha>0\}, I^{-1}(\alpha)=\alpha^{2}$ and

$$
\hat{\alpha}_{n}=1 / \overline{X_{n}^{2}}, \quad \overline{X_{n}^{2}}=\frac{1}{n} \sum_{j=1}^{n} X_{j}^{2} .
$$

The quantities $a_{n 1}^{(r, k)}, b_{n 1}^{(r, k)}, c_{n 1}^{(r, k)}$ are as for the exponential distribution and the tests statistics are given by similar formulae. 
5.3. Normal distribution: $X \sim N\left(\mu, \sigma^{2}\right)$. Here by $f$ and $F$ we denote the corresponding density and the cumulative distribution function, $\Lambda=\left\{\left(\mu, \sigma^{2}\right): \mu \in \mathbb{R}, \sigma^{2}>0\right\}$, and

$$
\hat{\mu}_{n}=\bar{X}_{n}, \quad \hat{\sigma}_{n}^{2}=\frac{1}{n} \sum_{i=1}^{n}\left(X_{i}-\bar{X}_{n}\right)^{2}, \quad S_{n}=\sqrt{\hat{\sigma}_{n}^{2}}, \quad \mathcal{I}^{-1}=\left[\begin{array}{cc}
\sigma^{2} & 0 \\
0 & 2 \sigma^{4}
\end{array}\right] .
$$

Hence

$$
\begin{aligned}
b^{(r, k)}(\mu) & =E\left[\left(\bar{F}\left(X ; \mu, \sigma^{2}\right)\right)^{k-2} \log ^{r} \frac{1}{\bar{F}\left(X ; \mu, \sigma^{2}\right)} \frac{\partial F\left(X ; \mu, \sigma^{2}\right)}{\partial \mu}\right] \\
& =-\frac{1}{\sigma} E\left[(\bar{\Phi}(Z))^{k-2} \log ^{r} \frac{1}{\bar{\Phi}(Z)} \phi(Z)\right]=-\frac{1}{\sigma} E_{1}^{(r, k)}
\end{aligned}
$$

where

$$
E_{1}^{(r, k)}=E\left[\phi(Z)(\bar{\Phi}(Z))^{k-2} \log ^{r} \frac{1}{\bar{\Phi}(Z)}\right], \quad Z \sim N(0,1),
$$

and $\phi, \Phi$ denote the pdf and cdf of $Z$, respectively. Similarly

$$
b^{(r, k)}\left(\sigma^{2}\right)=-\frac{1}{2 \sigma^{2}} E_{2}^{(r, k)}, \quad \text { where } \quad E_{2}^{(r, k)}=E\left[Z \phi(Z)(\bar{\Phi}(Z))^{k-2} \log ^{r} \frac{1}{\bar{\Phi}(Z)}\right] .
$$

Then

$$
\boldsymbol{B}^{(r, k)}=\left[\begin{array}{cc}
-\frac{r+2}{\sigma} E_{1}^{(r+1, k)}+\frac{k-1}{\sigma} E_{1}^{(r+2, k)} & -\frac{r+2}{2 \sigma^{2}} E_{2}^{(r+1, k)}+\frac{k-1}{2 \sigma^{2}} E_{2}^{(r+2, k)} \\
-\frac{k(r+1)}{\sigma} E_{1}^{(r, k)} & -\frac{k(r+1)}{2 \sigma^{2}} E_{2}^{(r, k)}
\end{array}\right]
$$

and

$$
\boldsymbol{K}_{n}^{(r, k)}=\frac{1}{n} \boldsymbol{B}^{(r, k)} \mathcal{I}^{-1}\left(\boldsymbol{B}^{(r, k)}\right)^{\prime}=\left[\begin{array}{cc}
s_{n}^{(r)} & t_{n}^{(r)} \\
t_{n}^{(r)} & u_{n}^{(r)}
\end{array}\right]
$$

with

$$
\begin{aligned}
s_{n}^{(r, k)}= & \frac{1}{n}\left[\left[(k-1) E_{1}^{(r+2, k)}-(r+2) E_{1}^{(r+1, k)}\right]^{2}+\frac{1}{2}\left[(k-1) E_{2}^{(r+2, k)}-(r+2) E_{2}^{(r+1, k)}\right]^{2}\right], \\
t_{n}^{(r, k)=} & \frac{k(r+1)}{n}\left[(r+2)\left(E_{1}^{(r, k)} E_{1}^{(r+1, k)}+\frac{1}{2} E_{2}^{(r, k)} E_{2}^{(r+1, k)}\right)\right. \\
& \left.-(k-1)\left(E_{1}^{(r, k)} E_{1}^{(r+2, k)}+\frac{1}{2} E_{2}^{(r, k)} E_{2}^{(r+1, k)}\right)\right], \\
u_{n}^{(r, k)}= & \frac{k^{2}(r+1)^{2}}{n}\left[\left(E_{1}^{(r, k)}\right)^{2}+\frac{1}{2}\left(E_{2}^{(r, k)}\right)^{2}\right], \\
a_{n 1}^{(r, k)}= & a_{n}^{(r, k)}-\frac{1}{n}\left[\left((k-1) E_{1}^{(r+2, k)}-(r+2) E_{1}^{(r+1, k)}\right)^{2}\right. \\
& \left.+\frac{1}{2}\left((k-1) E_{2}^{(r+2, k)}-(r+2) E_{2}^{(r+1, k)}\right)^{2}\right], \\
b_{n 1}^{(r, k)}= & b_{n}^{(r, k)}-\frac{k(r+1)}{n}\left[(r+2)\left(E_{1}^{(r, k)} E_{1}^{(r+1, k)}+\frac{1}{2} E_{2}^{(r, k)} E_{2}^{(r+1, k)}\right)\right. \\
& \left.-(k-1)\left(E_{1}^{(r, k)} E_{1}^{(r+2, k)}+\frac{1}{2} E_{2}^{(r, k)} E_{2}^{(r+1, k)}\right)\right], \\
c_{n 1}^{(r, k)}= & c_{n}^{(r, k)}-\frac{k^{2}(r+1)^{2}}{n}\left[\left(E_{1}^{(r, k)}\right)^{2}+\frac{1}{2}\left(E_{2}^{(r, k)}\right)^{2}\right] .
\end{aligned}
$$


The test-statistics for the direct tests are

$$
\begin{aligned}
\hat{T}_{n}^{(r, k)=} & \frac{1}{\Delta_{n 1}^{(r, k)}}\left[c_{n 1}^{(r, k)}\left(\frac{1}{n} \sum_{i=1}^{n}\left(\bar{\Phi}\left(\frac{X_{i}-\bar{X}_{n}}{S_{n}}\right)\right)^{k-1}\left|\log \bar{\Phi}\left(\frac{X_{i}-\bar{X}_{n}}{S_{n}}\right)\right|^{r+2}-\frac{\Gamma(r+3)}{k^{r+3}}\right)^{2}\right. \\
& -2 b_{n 1}^{(r, k)}\left(\frac{1}{n} \sum_{i=1}^{n}\left(\bar{\Phi}\left(\frac{X_{i}-\bar{X}_{n}}{S_{n}}\right)\right)^{k-1}\left|\log \bar{\Phi}\left(\frac{X_{i}-\bar{X}_{n}}{S_{n}}\right)\right|^{r+2}-\frac{\Gamma(r+3)}{k^{r+3}}\right) \\
& \cdot\left(\frac{1}{\left(\begin{array}{l}
n \\
k
\end{array}\right)} \sum_{i=1}^{n-k+1}\left(\begin{array}{l}
n-i \\
k-1
\end{array}\right)\left|\log \bar{\Phi}\left(\frac{X_{i: n}-\bar{X}_{n}}{S_{n}}\right)\right|^{r+1}-\frac{\Gamma(r+2)}{k^{r+1}}\right) \\
& \left.+a_{n 1}^{(r, k)}\left(\frac{1}{\left(\begin{array}{l}
n \\
k
\end{array}\right)} \sum_{i=1}^{n-k+1}\left(\begin{array}{l}
n-i \\
k-1
\end{array}\right)\left|\log \bar{\Phi}\left(\frac{X_{i: n}-\bar{X}_{n}}{S_{n}}\right)\right|^{r+1}-\frac{\Gamma(r+2)}{k^{r+1}}\right)^{2}\right] \\
= & \hat{T}_{n ; c_{1}}^{(r, k)}+\hat{T}_{n ; c_{2}}^{(r, k)}=\hat{T}_{n ; c_{3}}^{(r, k)}+\hat{T}_{n ; c_{4}}^{(r, k)}
\end{aligned}
$$

where

$$
\begin{aligned}
& \hat{T}_{n ; c_{1}}^{(r, k)}=\left[\frac{1}{n} \sum_{i=1}^{n}\left(\bar{\Phi}\left(\frac{X_{i}-\bar{X}_{n}}{S_{n}}\right)\right)^{k-1}\left|\log \bar{\Phi}\left(\frac{X_{i}-\bar{X}_{n}}{S_{n}}\right)\right|^{r+2}-\frac{\Gamma(r+3)}{k^{r+3}}\right]^{2}, \\
& \hat{T}_{n ; c_{2}}^{(r, k)}=\frac{1}{\Delta_{n 1}^{(r, k)} a_{n 1}^{(r, k)}}\left[a_{n 1}^{(r, k)} \frac{1}{\left(\begin{array}{l}
n \\
k
\end{array}\right)} \sum_{i=1}^{n-k+1}\left(\begin{array}{c}
n-i \\
k-1
\end{array}\right)\left|\log \bar{\Phi}\left(\frac{X_{i: n}-\bar{X}_{n}}{S_{n}}\right)\right|^{r+1}\right. \\
& -b_{n 1}^{(r, k)} \frac{1}{n} \sum_{i=1}^{n}\left(\bar{\Phi}\left(\frac{X_{i}-\bar{X}_{n}}{S_{n}}\right)\right)^{k-1}\left|\log \bar{\Phi}\left(\frac{X_{i}-\bar{X}_{n}}{S_{n}}\right)\right|^{r+2} \\
& \left.-\frac{\Gamma(r+2)}{k^{r+3}}\left(k^{2} a_{n 1}^{(r, k)}-(r+2) b_{n 1}^{(r, k)}\right)\right]^{2} \text {, } \\
& \hat{T}_{n ; c_{3}}^{(r, k)}=\frac{1}{c_{n 1}^{(r, k)}}\left[\frac{1}{\left(\begin{array}{l}
n \\
k
\end{array}\right)} \sum_{i=1}^{n-k+1}\left(\begin{array}{l}
n-i \\
k-1
\end{array}\right)\left|\log \bar{\Phi}\left(\frac{X_{i: n}-\bar{X}_{n}}{S_{n}}\right)\right|^{r+1}-\frac{\Gamma(r+2)}{k^{r+1}}\right]^{2}, \\
& \hat{T}_{n ; c_{4}}^{(r, k)}=\frac{1}{\Delta_{n 1}^{(r, k)} c_{n 1}^{(r, k)}}\left[c_{n 1}^{(r, k)} \frac{1}{n} \sum_{i=1}^{n}\left(\bar{\Phi}\left(\frac{X_{i}-\bar{X}_{n}}{S_{n}}\right)\right)^{k-1} \cdot\left|\log \bar{\Phi}\left(\frac{X_{i}-\bar{X}_{n}}{S_{n}}\right)\right|^{r+2}\right. \\
& \left.-\frac{b_{n 1}^{(r, k)}}{\left(\begin{array}{l}
n \\
k
\end{array}\right)} \sum_{i=1}^{n-k+1}\left(\begin{array}{l}
n-i \\
k-1
\end{array}\right)\left|\log \Phi\left(\frac{X_{i: n}-\bar{X}_{n}}{S_{n}}\right)\right|^{r+1}-\frac{\Gamma(r+2)}{k^{r+3}}\left((r+2) c_{n 1}^{(r, k)}-k^{2} b_{n 1}^{(r, k)}\right)\right]^{2} .
\end{aligned}
$$

6. Simulations. We have selected tests and alternatives in Table 1 from Cabaña and Cabaña [6] as standards of comparison with our proposed tests. When $n=20$ and $n=50$ the test-statistics $\hat{T}_{n}^{(r, k)}$ and their components for $r=-0.9,-0.5,-0.3,-0.1,0.1,0.3,0.5$, $0.9,1.0,1.2,1.5,1.7,2.0$ and $k=1,2,3,4,5$ and 6 (not all for all tests). Critical values were simulated using 100000 and the associated powers were obtained using 100000 samples, but only some results are presented here.

For samples of size 20 we include simulations for some favorable omnibus tests with Av. powers $\geq 44.5$ (Table 2). Powers of tests with Av. powers $<44.5$ and greatest powers 
for some alternatives are given in Table 3. For samples of size 50 we include (Table 4) simulations for tests with Av. powers $\geq 75.2$. Table 5 contains tests with Av. powers $<75.2$ and greatest powers for some alternatives. Bold numbers stand for powers greater than those from Table 1, and the stars denote the overall maxima.

Table 1. (Source: Cabaña and Cabaña [6]) Empirical comparison of the performances of the test TEEP based on 100000 replications and of seven other tests, under several alternatives. The entries are simulated powers of $5 \%$ tests.

\begin{tabular}{|c|c|c|c|c|c|c|c|c|c|}
\hline \multirow{19}{*}{$n=20$} & Alt. & $T E E P$ & $E P$ & $B H K S$ & $B H C M$ & $S$ & $\mathrm{CO}$ & $B H$ & $T$ \\
\hline & $W(0.8)$ & 26 & 24 & 17 & 22 & 24 & 28 & 24 & 1 \\
\hline & $W(1.4)$ & 35 & 36 & 28 & 35 & 35 & 37 & 37 & 45 \\
\hline & $\Gamma(0.4)$ & 85 & 76 & 71 & 75 & 76 & 91 & 80 & 11 \\
\hline & $\Gamma(2)$ & 54 & 48 & 40 & 47 & 46 & 54 & 51 & 56 \\
\hline & $L N(0.8)$ & 37 & 25 & 30 & 27 & 24 & 33 & 29 & 34 \\
\hline & $L N(1.5)$ & 64 & 67 & 58 & 66 & 67 & 60 & 66 & 2 \\
\hline & $H N$ & 17 & 21 & 18 & 22 & 21 & 19 & 21 & $31 *$ \\
\hline & $U$ & 47 & 66 & 52 & 70 & 70 & 50 & 61 & 82 \\
\hline & $C H(0.5)$ & 72 & 63 & 56 & 61 & 63 & 80 & 67 & 6 \\
\hline & $C H(1)$ & 12 & 15 & 13 & 16 & 15 & 13 & 15 & $23^{*}$ \\
\hline & $C H(1.5)$ & 77 & 84 & 67 & 83 & 84 & 81 & 83 & $89 *$ \\
\hline & $L F(2)$ & 24 & 28 & 24 & 30 & 29 & 25 & 25 & $39 *$ \\
\hline & $L F(4)$ & 36 & 42 & 34 & 43 & 42 & 37 & 41 & $54^{*}$ \\
\hline & $E V(0.5)$ & 11 & 13 & 18 & 16 & 15 & 13 & 15 & $23^{*}$ \\
\hline & $E V(1.5)$ & 35 & 35 & 48 & 47 & 46 & 37 & 43 & $58^{*}$ \\
\hline & $D L(1)$ & 25 & 20 & 20 & 21 & 19 & 25 & 23 & 28 \\
\hline & $D L(1.5)$ & 72 & 64 & 56 & 63 & 62 & 72 & 68 & 71 \\
\hline & Av & 42.9 & 42.8 & 38.2 & 43.8 & 43.4 & 44.4 & 44.1 & 38.4 \\
\hline \multirow{18}{*}{$n=50$} & $W(0.8)$ & 53 & 48 & 35 & 46 & 48 & 56 & 50 & 17 \\
\hline & $W(1.4)$ & 82 & 80 & 71 & 77 & 79 & 82 & 81 & 81 \\
\hline & $\Gamma(0.4)$ & $100 *$ & 99 & 97 & 99 & 98 & $100 *$ & 99 & 90 \\
\hline & $\Gamma(2)$ & 94 & 91 & 86 & 90 & 90 & $96 *$ & 93 & 92 \\
\hline & $L N(0.8)$ & 73 & 45 & 62 & 60 & 47 & 66 & 58 & 66 \\
\hline & $L N(1.5)$ & 95 & 95 & 92 & 95 & 95 & 92 & 95 & 54 \\
\hline & $H N$ & 45 & 54 & 50 & 53 & 54 & 45 & 52 & $60^{*}$ \\
\hline & $U$ & 93 & 98 & 99 & 99 & 99 & 91 & 97 & $100^{*}$ \\
\hline & $C H(0.5)$ & 98 & 94 & 90 & 94 & 94 & $99 *$ & 96 & 79 \\
\hline & $C H(1)$ & 31 & 38 & 36 & 37 & 38 & 30 & 35 & $44^{*}$ \\
\hline & $C H(1.5)$ & $100 *$ & $100 *$ & $100^{*}$ & $100^{*}$ & $100 *$ & $100 *$ & $100 *$ & $100^{*}$ \\
\hline & $L F(2)$ & 61 & 69 & 65 & 69 & 69 & 60 & 68 & $74^{*}$ \\
\hline & $L F(4)$ & 81 & 87 & 82 & 87 & 87 & 80 & 86 & $90^{*}$ \\
\hline & $E V(0.5)$ & 30 & 38 & 36 & 37 & 38 & 30 & 35 & $44^{*}$ \\
\hline & $E V(1.5)$ & 80 & 90 & 88 & 90 & 90 & 78 & 87 & 93 \\
\hline & $D L(1)$ & 57 & 39 & 43 & 44 & 39 & 55 & 47 & 54 \\
\hline & $D L(1.5)$ & 99 & 97 & 96 & 97 & 97 & 99 & 98 & 98 \\
\hline & $\mathrm{Av}$ & 74.8 & 74.2 & 72.2 & 74.9 & 74.2 & 74.1 & 75.1 & 72.7 \\
\hline
\end{tabular}

The meaning of the headings and the test-statistics can be found in Cabaña and Cabaña [6] and Henze and Meintanis [11] and their references:

TEEP: The transformed estimated empirical process (TEEP) test EP: The Epps and Pulley test

BHKS: The Baringhaus and Henze test suggested by the Kolmogorov-Smirnov statistic BHCM: The Baringhaus and Henze test suggested by the Cramér-von Mises statistic

$S$ : The test based on spacing

$C O$ : The Cox and Oakes test

$B H$ : The Baringhaus and Henze test based on the empirical Laplace transform

T: The Henze and Meintanis test based on the empirical characteristic function (ECF). 
The alternatives considered are:

$W(\theta)$ - Weibull distribution with parameters $(1, \theta)$

$\Gamma(\theta)$ - Gamma distribution with parameters $(1, \theta)$

$L N(\theta)$ - Lognormal distribution with parameters $(0, \theta)$

$H N$ - Half-normal distribution: the law of $|Z|, Z$ standard normal

$U$ - Uniform distribution on $[0,1]$

$E V(\theta)$ - Modified extreme value distribution: the law of $\log (1-\theta \log U), U$ uniform on $[0,1]$

$L F(\theta)$ - Linear increasing failure rate: the law of $\theta^{-1}(\sqrt{1+2 Y \theta}-1), Y \sim \operatorname{Exp}(1)$

$D L(\theta)$ - Dhillon's Distribution: the law of $e^{(-\log U)^{1 /(\theta+1)}}-1, U$ uniform on $[0,1]$

$C H(\theta)$ - Chen Distribution: the law of $\left(\log \left(1-\frac{1}{2} \log U\right)\right)^{1 / \theta}, U$ uniform on [0,1].

Table 2. Powers of $5 \%$ tests based on 100000 simulations using empirical critical values with Av. powers $\geq 44.5 ; n=20$

\begin{tabular}{|c|cc|ccccc|cc|}
\hline$k$ & \multicolumn{3}{|c|}{1} & \multicolumn{3}{c|}{2} & \multicolumn{2}{c|}{3} \\
\hline Tests & $\hat{T}_{n ; c_{2}}^{(r, k)}$ & $\hat{T}_{n ; c_{3}}^{(r, k)}$ & $\hat{T}_{n ; c_{3}}^{(r, k)}$ & $\hat{T}_{n}^{(r, k)}$ & $\hat{T}_{n ; c_{3}}^{(r, k)}$ & $\hat{T}_{n}^{(r, k)}$ & $\hat{T}_{n ; c_{3}}^{(r, k)}$ & $\hat{T}_{n ; c_{3}}^{(r, k)}$ & $\hat{T}_{n ; c_{3}}^{(r, k)}$ \\
\hline Alt $\backslash r$ & -0.3 & -0.3 & -0.5 & -0.3 & -0.3 & -0.1 & -0.1 & -0.5 & -0.3 \\
\hline$W(0.8)$ & 26 & 26 & 26 & 26 & 25 & 25 & 24 & 24 & 22 \\
$W(1.4)$ & 38 & 37 & 39 & 37 & 39 & 36 & 37 & 39 & 41 \\
$G(0.4)$ & 84 & 87 & 88 & 87 & 84 & 82 & 79 & 89 & 86 \\
$G(2)$ & 53 & 51 & 55 & 51 & 54 & 49 & 50 & $\mathbf{5 8}$ & $\mathbf{5 9}$ \\
$L N(0.8)$ & 31 & 30 & 36 & 32 & 32 & 28 & 27 & $\mathbf{4 5}$ & $\mathbf{4 4}$ \\
$L N(1.5)$ & 65 & 66 & 59 & 63 & 62 & 66 & 65 & 48 & 52 \\
$H N$ & 20 & 20 & 20 & 21 & 21 & 21 & 22 & 19 & 21 \\
$U$ & 59 & 60 & 57 & 67 & 65 & 71 & 69 & 48 & 55 \\
$C H(0.5)$ & 72 & 70 & 77 & 75 & 72 & 69 & 66 & 78 & 74 \\
$C H(1)$ & 14 & 13 & 14 & 14 & 15 & 15 & 15 & 13 & 15 \\
$C H(1.5)$ & 83 & 83 & 82 & 83 & 84 & 84 & 85 & 78 & 82 \\
$L F(2)$ & 28 & 28 & 28 & 29 & 30 & 30 & 30 & 26 & 29 \\
$L F(4)$ & 41 & 41 & 41 & 43 & 44 & 44 & 44 & 39 & 43 \\
$E V(0.5)$ & 14 & 14 & 14 & 14 & 15 & 15 & 15 & 13 & 15 \\
$E V(1.5)$ & 42 & 42 & 41 & 45 & 45 & 47 & 47 & 37 & 41 \\
$D L(1)$ & 24 & 23 & 26 & 23 & 24 & 21 & 21 & $\mathbf{3 1}$ & $\mathbf{3 1}$ \\
$D L(1.5)$ & 70 & 68 & $\mathbf{7 3}$ & 68 & 71 & 65 & 66 & $\mathbf{7 8}$ & $\mathbf{7 8}$ \\
\hline Av. & 44.9 & 44.5 & 45.7 & 45.8 & 45.9 & 45.0 & 44.9 & 44.9 & 46.3 \\
\hline
\end{tabular}

\begin{tabular}{|c|c|c|c|c|c|c|c|c|c|}
\hline \multirow{2}{*}{$\begin{array}{c}k \\
\text { Tests }\end{array}$} & \multicolumn{4}{|c|}{3} & \multicolumn{5}{|c|}{4} \\
\hline & $\hat{T}_{n ; c_{3}}^{(r, k)}$ & $\hat{T}_{n ; c_{3}}^{(r, k)}$ & $\hat{T}_{n ; c}^{(r, k)}$ & $\hat{T}_{n ; c_{3}}^{(r, k)}$ & $\hat{T}_{n ; c_{2}}^{(r, k)}$ & $\hat{T}_{n ; c_{3}}^{(r, k)}$ & $\hat{T}_{n ; c_{3}}^{(r, k)}$ & $\hat{T}_{n ; c_{3}}^{(r, k)}$ & $\hat{T}_{n ; c}^{(r, k)}$ \\
\hline Alt $\backslash r$ & -0.1 & 0.1 & 0.3 & 0.5 & -0.5 & -0.3 & -0.1 & 0.1 & 0.3 \\
\hline$W(0.8)$ & 21 & 20 & 20 & 20 & 18 & 19 & 18 & 16 & 16 \\
\hline$W(1.4)$ & 42 & 42 & 40 & 39 & 43 & 42 & 43 & 44 & 45 \\
\hline$G(0.4)$ & 82 & 78 & 74 & 70 & 76 & 86 & 83 & 79 & 76 \\
\hline$G(2)$ & 59 & 57 & 54 & 51 & 58 & 61 & 62 & 62 & 62 \\
\hline$L N(0.8)$ & 40 & 36 & 32 & 28 & 35 & 52 & 50 & 47 & 44 \\
\hline$L N(1.5)$ & 55 & 58 & 60 & 62 & 41 & 41 & 43 & 46 & 49 \\
\hline$H N$ & 23 & 23 & 24 & 24 & 24 & 20 & 22 & 24 & 25 \\
\hline$U$ & 60 & 65 & 69 & 72 & 69 & 48 & 53 & 58 & 62 \\
\hline$C H(0.5)$ & 69 & 64 & 61 & 57 & 61 & 73 & 69 & 64 & 60 \\
\hline $\mathrm{CH}(1)$ & 16 & 16 & 17 & 17 & 17 & 14 & 16 & 17 & 18 \\
\hline $\mathrm{CH}(1.5)$ & 84 & 84 & 85 & 85 & 87 & 79 & 81 & 83 & 84 \\
\hline$L F(2)$ & 31 & 32 & 33 & 33 & 33 & 28 & 30 & 32 & 34 \\
\hline$L F(4)$ & 45 & 46 & 47 & 47 & 47 & 40 & 43 & 46 & 48 \\
\hline$E V(0.5)$ & 16 & 17 & 17 & 17 & 17 & 15 & 16 & 17 & 18 \\
\hline$E V(1.5)$ & 44 & 47 & 49 & 50 & 49 & 37 & 41 & 44 & 47 \\
\hline$D L(1)$ & 29 & 27 & 25 & 22 & 27 & 35 & 34 & 33 & 33 \\
\hline$D L(1.5)$ & 76 & 74 & 71 & 67 & 74 & 80 & 80 & 80 & 79 \\
\hline Av. & 46.7 & 46.2 & 45.8 & 44.7 & 45.8 & 45.3 & 46.1 & 46.6 & 47.0 \\
\hline
\end{tabular}




\begin{tabular}{|c|cccc|cccc|}
\hline$k$ & \multicolumn{5}{|c}{4} & & \multicolumn{3}{|c|}{5} \\
\hline Tests & $\hat{T}_{n ; c_{3}}^{(r, k)}$ & $\hat{T}_{n ; c_{3}}^{(r, k)}$ & $\hat{T}_{n ; c_{3}}^{(r, k)}$ & $\hat{T}_{n ; c_{3}}^{(r, k)}$ & $\hat{T}_{n ; c_{2}}^{(r, k)}$ & $\hat{T}_{n}^{(r, k)}$ & $\hat{T}_{n ; c_{2}}^{(r, k)}$ & $\hat{T}_{n ; c_{3}}^{(r, k)}$ \\
\hline Alt $\backslash r$ & 0.5 & 0.75 & 0.9 & 1.0 & -0.5 & -0.3 & -0.3 & -0.1 \\
\hline$W(0.8)$ & 15 & 15 & 15 & 15 & 17 & 15 & 13 & 14 \\
$W(1.4)$ & 44 & 43 & 43 & 42 & $\mathbf{4 6}$ & 39 & $\mathbf{4 8}$ & 44 \\
$G(0.4)$ & 71 & 69 & 65 & 64 & 83 & 82 & 68 & 81 \\
$G(2)$ & $\mathbf{6 0}$ & $\mathbf{5 8}$ & 56 & 55 & $\mathbf{6 4}$ & $\mathbf{5 8}$ & $\mathbf{6 5}$ & $\mathbf{6 4}$ \\
$L N(0.8)$ & $\mathbf{4 1}$ & 36 & 34 & 32 & $\mathbf{4 8}$ & $\mathbf{5 6}$ & $\mathbf{4 7}$ & $\mathbf{5 7}$ \\
$L N(1.5)$ & 50 & 53 & 55 & 56 & 38 & 33 & 37 & 32 \\
$H N$ & 26 & 27 & 27 & 27 & 24 & 20 & 26 & 21 \\
$U$ & 65 & 68 & 70 & 71 & 58 & 59 & 64 & 47 \\
$C H(0.5)$ & 56 & 53 & 51 & 50 & 68 & 66 & 52 & 66 \\
$C H(1)$ & 19 & 19 & 19 & 19 & 17 & 15 & 19 & 16 \\
$C H(1.5)$ & 85 & 85 & 85 & 85 & 85 & 80 & 87 & 79 \\
$L F(2)$ & 35 & 35 & 36 & 35 & 32 & 27 & 35 & 29 \\
$L F(4)$ & 49 & 50 & 50 & 50 & 46 & 40 & 49 & 42 \\
$E V(0.5)$ & 18 & 19 & 19 & 19 & 17 & 15 & 19 & 15 \\
$E V(1.5)$ & 49 & 50 & 51 & 52 & 44 & 40 & 49 & 38 \\
$D L(1)$ & $\mathbf{3 0}$ & 28 & 26 & 25 & $\mathbf{3 5}$ & $\mathbf{3 4}$ & $\mathbf{3 5}$ & $\mathbf{3 8}$ \\
$D L(1.5)$ & $\mathbf{7 7}$ & $\mathbf{7 4}$ & 72 & 71 & $\mathbf{8 2}$ & $\mathbf{7 7}$ & $\mathbf{8 2}$ & $\mathbf{8 2}$ \\
\hline Av. & 46.5 & 45.9 & 45.5 & 45.2 & $\mathbf{4 7 . 2}$ & 44.5 & 46.8 & 45.0 \\
\hline
\end{tabular}

\begin{tabular}{|c|c|c|c|c|c|c|c|c|}
\hline$k$ & \multicolumn{6}{|c|}{5} & \multicolumn{2}{|c|}{6} \\
\hline Tests & $\hat{T}_{n ; c_{3}}^{(r, k)}$ & $\hat{T}_{n ; c_{3}}^{(r, k)}$ & $\hat{T}_{n ; c_{3}}^{(r, k)}$ & $\hat{T}_{n ; c_{3}}^{(r, k)}$ & $\hat{T}_{n ; c_{3}}^{(r, k)}$ & $\hat{T}_{n ; c_{3}}^{(r, k)}$ & $\hat{T}_{n ; c_{2}}^{(r, k)}$ & $\hat{T}_{n ; c_{2}}^{(r, k)}$ \\
\hline Alt $\backslash r$ & 0.1 & 0.3 & 0.5 & 0.75 & 0.9 & 1.0 & -0.5 & -0.3 \\
\hline$W(0.8)$ & 12 & 11 & 9 & 8 & 8 & 8 & 16 & 12 \\
\hline$W(1.4)$ & 45 & 46 & 47 & 47 & 47 & 46 & 44 & 47 \\
\hline$G(0.4)$ & 77 & 73 & 67 & 60 & 58 & 56 & 86 & 76 \\
\hline$G(2)$ & 64 & 65 & 64 & 63 & 63 & 62 & 65 & 67 \\
\hline$L N(0.8)$ & 55 & 53 & 50 & 47 & 45 & 43 & 56 & 56 \\
\hline$L N(1.5)$ & 33 & 35 & 35 & 36 & 37 & 38 & 30 & 31 \\
\hline$H N$ & 23 & 25 & 26 & 27 & 28 & 28 & 21 & 24 \\
\hline$U$ & 52 & 56 & 60 & 63 & 65 & 66 & 47 & 54 \\
\hline $\mathrm{CH}(0.5)$ & 60 & 56 & 50 & 44 & 42 & 41 & 71 & 59 \\
\hline $\mathrm{CH}(1)$ & 17 & 18 & 19 & 20 & 20 & 20 & 15 & 17 \\
\hline $\mathrm{CH}(1.5)$ & 81 & 83 & 84 & 85 & 85 & 86 & 79 & 83 \\
\hline$L F(2)$ & 31 & 34 & 34 & 36 & 37 & 37 & 29 & 32 \\
\hline$L F(4)$ & 44 & 47 & 48 & 50 & 51 & 51 & 41 & 45 \\
\hline$E V(0.5)$ & 17 & 18 & 19 & 19 & 20 & 20 & 15 & 18 \\
\hline$E V(1.5)$ & 41 & 44 & 46 & 48 & 50 & 51 & 38 & 42 \\
\hline$D L(1)$ & 38 & 38 & 36 & 34 & 34 & 33 & 38 & 39 \\
\hline$D L(1.5)$ & 82 & 82 & 82 & 80 & 79 & 78 & 83 & $84^{*}$ \\
\hline Av. & 45.4 & 46.1 & 45.7 & 45.0 & 45.1 & 45.0 & 45.5 & 46.4 \\
\hline
\end{tabular}

Table 3. Tests with Av. powers $<44.5$ and greatest powers for some alternatives; $n=20$

\begin{tabular}{|c|c|c|c|c|c|c|c|c|c|c|}
\hline$k$ & \multicolumn{2}{|c|}{5} & \multicolumn{8}{|c|}{6} \\
\hline Tests & $\hat{T}_{n ; c_{2}}^{(r, k)}$ & $\hat{T}_{n ; c_{2}}^{(r, k)}$ & $\hat{T}_{n ; c 3}^{(r, k)}$ & $\hat{T}_{n ; c_{2}}^{(r, k)}$ & $\hat{T}_{n ; c_{3}}^{(r, k)}$ & $\hat{T}_{n ; c_{2}}^{(r, k)}$ & $\hat{T}_{n ; c}^{(r, k)}$ & $\hat{T}_{n ; c}^{(r, k)}$ & $\hat{T}_{n ; c}^{(r, k)}$ & $\hat{T}_{n ; c_{2}}^{(r, k)}$ \\
\hline Alt $\backslash r$ & 0.1 & 0.3 & -0.3 & -0.1 & -0.1 & 0.1 & 0.1 & 0.3 & 0.3 & 0.5 \\
\hline$W(0.8)$ & 8 & 7 & 14 & 7 & 11 & 5 & 8 & 4 & 5 & 3 \\
\hline$W(1.4)$ & 47 & 46 & 41 & 48 & 43 & $49 *$ & 45 & $49^{*}$ & 46 & $49 *$ \\
\hline$G(0.4)$ & 20 & 12 & 84 & 47 & 79 & 20 & 72 & 10 & 64 & 5 \\
\hline$G(2)$ & 63 & 61 & 63 & $68^{*}$ & 65 & 67 & 66 & 67 & 67 & 65 \\
\hline$L N(0.8)$ & 42 & 39 & $62 *$ & 54 & $62 *$ & 52 & 61 & 49 & 59 & 46 \\
\hline$L N(1.5)$ & 26 & 21 & 21 & 27 & 21 & 21 & 20 & 17 & 19 & 13 \\
\hline$H N$ & 28 & 28 & 19 & 26 & 21 & 27 & 22 & 28 & 24 & 29 \\
\hline$U$ & 71 & 74 & 39 & 58 & 43 & 62 & 47 & 66 & 51 & 69 \\
\hline $\mathrm{CH}(0.5)$ & 16 & 10 & 69 & 34 & 62 & 15 & 53 & 8 & 45 & 4 \\
\hline$C H(1)$ & 20 & 20 & 14 & 19 & 15 & 19 & 16 & 21 & 17 & 21 \\
\hline $\mathrm{CH}(1.5)$ & $89 *$ & $89 *$ & 74 & 85 & 77 & 86 & 79 & 87 & 81 & 88 \\
\hline$L F(2)$ & 37 & 38 & 25 & 34 & 28 & 36 & 30 & 38 & 32 & 38 \\
\hline$L F(4)$ & 52 & 52 & 37 & 48 & 40 & 50 & 42 & 42 & 45 & 52 \\
\hline$E V(0.5)$ & 20 & 21 & 14 & 18 & 15 & 19 & 16 & 21 & 18 & 21 \\
\hline$E V(1.5)$ & 53 & 54 & 32 & 45 & 36 & 48 & 39 & 51 & 41 & 52 \\
\hline$D L(1)$ & 32 & 30 & 39 & 39 & $41 *$ & 37 & $41 *$ & 37 & $41 *$ & 35 \\
\hline$D L(1.5)$ & 79 & 76 & 82 & $84^{*}$ & 83 & 83 & $84^{*}$ & 83 & $84 *$ & 81 \\
\hline Av. & 41.3 & 39.9 & 42.8 & 43.6 & 43.6 & 41.0 & 43.5 & 40.4 & 43.6 & 39.5 \\
\hline
\end{tabular}


Table 4. Powers of $5 \%$ tests based on 100000 simulations using empirical critical values with Av. powers $\geq 75.2 ; n=50$

\begin{tabular}{|c|c|c|c|c|c|c|c|c|c|c|}
\hline$k$ & 1 & \multicolumn{6}{|c|}{2} & \multicolumn{3}{|c|}{3} \\
\hline Tests & $\hat{T}_{n ; c_{2}}^{(r, k)}$ & $\hat{T}_{n}^{(r, k)}$ & $\hat{T}_{n ; c_{3}}^{(r, k)}$ & $\hat{T}_{n}^{(r, k)}$ & $\hat{T}_{n ; c_{3}}^{(r, k)}$ & $\hat{T}_{n}^{(r, k)}$ & $\hat{T}_{n ; c}^{(r, k)}$ & $\hat{T}_{n ; c}^{(r, k)}$ & $\hat{T}_{n ; c}^{(r, k)}$ & $\hat{T}_{n ; c}^{(r, k)}$ \\
\hline Alt $\backslash r$ & -0.3 & -0.5 & -0.5 & -0.3 & -0.3 & -0.1 & -0.1 & -0.3 & -0.1 & 0.1 \\
\hline$W(0.8)$ & 52 & 53 & 53 & 52 & 51 & 50 & 49 & 49 & 48 & 47 \\
\hline$W(1.4)$ & 82 & 78 & 82 & 80 & 82 & 80 & 80 & 82 & 82 & 82 \\
\hline$G(0.4)$ & $100 *$ & $100 *$ & $100 *$ & $100 *$ & $100 *$ & 99 & 99 & $100 *$ & $100 *$ & 99 \\
\hline$G(2)$ & 94 & 94 & 95 & 94 & 94 & 92 & 92 & 96 & 95 & 94 \\
\hline$L N(0.8)$ & 58 & 81 & 73 & 74 & 63 & 61 & 52 & 81 & 74 & 67 \\
\hline$L N(1.5)$ & 94 & 92 & 92 & 94 & 94 & 95* & $95 *$ & 89 & 91 & 93 \\
\hline$H N$ & 50 & 48 & 47 & 51 & 51 & 53 & 53 & 46 & 49 & 52 \\
\hline$U$ & 96 & 98 & 94 & 98 & 97 & 99 & 98 & 92 & 95 & 97 \\
\hline $\mathrm{CH}(0.5)$ & 98 & $99^{*}$ & 99* & 98 & 98 & 97 & 96 & 98 & 97 & 96 \\
\hline$C H(1)$ & 35 & 33 & 32 & 36 & 36 & 38 & 38 & 31 & 34 & 37 \\
\hline $\mathrm{CH}(1.5)$ & $100^{*}$ & $100 *$ & $100^{*}$ & $100 *$ & $100 *$ & $100 *$ & $100 *$ & $100^{*}$ & $100^{*}$ & $100^{*}$ \\
\hline$L F(2)$ & 67 & 64 & 64 & 68 & 68 & 70 & 70 & 62 & 66 & 68 \\
\hline$L F(4)$ & 85 & 83 & 83 & 86 & 86 & 87 & 87 & 82 & 84 & 86 \\
\hline$E V(0.5)$ & 35 & 32 & 32 & 36 & 35 & 38 & 38 & 31 & 34 & 36 \\
\hline$E V(1.5)$ & 86 & 86 & 82 & 89 & 87 & 90 & 90 & 80 & 83 & 87 \\
\hline$D L(1)$ & 50 & 58 & 59 & 54 & 52 & 47 & 45 & 65 & 60 & 55 \\
\hline$D L(1.5)$ & 99 & 99 & 99 & 99 & 99 & 98 & 98 & $100^{*}$ & 99 & 99 \\
\hline Av. & 75.3 & 76.3 & 75.7 & 77.0 & 76.0 & 76.1 & 75.2 & 75.4 & 76.0 & 76.2 \\
\hline
\end{tabular}

\begin{tabular}{|c|c|c|c|c|c|c|c|c|c|c|}
\hline$k$ & 3 & & & & & 4 & & & & \\
\hline Tests & $\hat{T}_{n ; c_{3}}^{(r, k)}$ & $\hat{T}_{n}^{(r, k)}$ & $\hat{T}_{n ; c_{2}}^{(r, k)}$ & $\hat{T}_{n}^{(r, k)}$ & $\hat{T}_{n}^{(r, k)}$ & $\hat{T}_{n ; c_{3}}^{(r, k)}$ & $\hat{T}_{n ; c 3}^{(r, k)}$ & $\hat{T}_{n ; c_{3}}^{(r, k)}$ & $\hat{T}_{n ; c_{3}}^{(r, k)}$ & $\hat{T}_{n ; c_{3}}^{(r, k)}$ \\
\hline Alt $\backslash r$ & 0.3 & -0.5 & -0.5 & -0.3 & -0.1 & 0.1 & 0.3 & 0.5 & 0.75 & 0.9 \\
\hline$W(0.8)$ & 46 & 47 & 44 & 45 & 43 & 45 & 43 & 43 & 42 & 41 \\
\hline$W(1.4)$ & 81 & 78 & 84 & 79 & 79 & 83 & 83 & 82 & 82 & 81 \\
\hline$G(0.4)$ & 98 & $100^{*}$ & 99 & $100^{*}$ & 99 & 99 & 99 & 99 & 98 & 97 \\
\hline$G(2)$ & 92 & 95 & 94 & 95 & 94 & 96 & 95 & 94 & 93 & 92 \\
\hline$L N(0.8)$ & 58 & 94 & 61 & 92 & 88 & 81 & 76 & 70 & 62 & 57 \\
\hline$L N(1.5)$ & 94 & 83 & 86 & 85 & 87 & 88 & 90 & 91 & 93 & 93 \\
\hline$H N$ & 54 & 42 & 55 & 44 & 45 & 48 & 51 & 53 & 55 & 56 \\
\hline$U$ & 98 & 97 & 98 & 98 & 98 & 93 & 95 & 97 & 98 & 98 \\
\hline $\mathrm{CH}(0.5)$ & 95 & $99 *$ & 96 & 98 & 97 & 97 & 96 & 95 & 93 & 91 \\
\hline$C H(1)$ & 38 & 29 & 40 & 30 & 31 & 33 & 35 & 37 & 39 & 40 \\
\hline $\mathrm{CH}(1.5)$ & $100^{*}$ & $100^{*}$ & $100^{*}$ & $100^{*}$ & $100^{*}$ & $100^{*}$ & $100^{*}$ & $100^{*}$ & $100^{*}$ & $100 *$ \\
\hline$L F(2)$ & 70 & 58 & 70 & 60 & 61 & 64 & 67 & 69 & 71 & 71 \\
\hline$L F(4)$ & 87 & 78 & 87 & 80 & 81 & 83 & 85 & 87 & 88 & 88 \\
\hline$E V(0.5)$ & 38 & 28 & 40 & 30 & 31 & 33 & 35 & 37 & 39 & 40 \\
\hline$E V(1.5)$ & 89 & 81 & 89 & 83 & 84 & 82 & 84 & 87 & 89 & 90 \\
\hline$D L(1)$ & 49 & 71 & 52 & 69 & 66 & 65 & 61 & 57 & 51 & 48 \\
\hline$D L(1.5)$ & 98 & $100 *$ & 99 & 99 & 99 & 99 & 99 & 99 & 99 & 98 \\
\hline Av. & 75.6 & 75.3 & 76.1 & 75.8 & 75.6 & 75.9 & 76.2 & 76.4 & 75.9 & 75.3 \\
\hline
\end{tabular}

\begin{tabular}{|c|c|c|c|c|c|c|c|c|c|c|}
\hline$k$ & \multicolumn{10}{|c|}{5} \\
\hline Tests & $\hat{T}_{n}^{(r, k)}$ & $\hat{T}_{n ; c_{2}}^{(r, k)}$ & $\hat{T}_{n}^{(r, k)}$ & $\hat{T}_{n ; c_{2}}^{(r, k)}$ & $\hat{T}_{n}^{(r, k)}$ & $\hat{T}_{n ; c_{2}}^{(r, k)}$ & $\hat{T}_{n}^{(r, k)}$ & $\hat{T}_{n}^{(r, k)}$ & $\hat{T}_{n ; c_{3}}^{(r, k)}$ & $\hat{T}_{n}^{(r, k)}$ \\
\hline Alt $\backslash r$ & -0.5 & -0.5 & -0.3 & -0.3 & -0.1 & -0.1 & 0.1 & 0.3 & 0.3 & 0.5 \\
\hline$W(0.8)$ & 44 & 47 & 41 & 43 & 39 & 37 & 36 & 34 & 40 & 32 \\
\hline$W(1.4)$ & 79 & 85 & 80 & 86 & 80 & 86 & 80 & 80 & 83 & 79 \\
\hline$G(0.4)$ & $100 *$ & $100 *$ & $100 *$ & 99 & 99 & 96 & 99 & 99 & 99 & 98 \\
\hline$G(2)$ & 95 & $97^{*}$ & 96 & $97^{*}$ & 95 & 96 & 95 & 94 & 96 & 93 \\
\hline$L N(0.8)$ & 96 & 82 & 95 & 78 & 94 & 72 & 92 & 89 & 85 & 86 \\
\hline$L N(1.5)$ & 82 & 85 & 83 & 87 & 83 & 87 & 84 & 84 & 85 & 84 \\
\hline$H N$ & 44 & 49 & 46 & 54 & 47 & 57 & 48 & 49 & 48 & 49 \\
\hline$U$ & 96 & 93 & 97 & 96 & 98 & 98 & 98 & 98 & 91 & 99 \\
\hline $\mathrm{CH}(0.5)$ & 98 & 98 & 98 & 96 & 97 & 89 & 96 & 94 & 96 & 92 \\
\hline$C H(1)$ & 30 & 34 & 31 & 38 & 33 & 41 & 34 & 34 & 32 & 34 \\
\hline $\mathrm{CH}(1.5)$ & $100 *$ & $100^{*}$ & $100^{*}$ & $100 *$ & $100 *$ & $100 *$ & $100^{*}$ & $100 *$ & $100 *$ & $100^{*}$ \\
\hline$L F(2)$ & 60 & 66 & 62 & 70 & 63 & 73 & 64 & 65 & 64 & 65 \\
\hline$L F(4)$ & 79 & 84 & 81 & 87 & 83 & 89 & 83 & 84 & 82 & 84 \\
\hline$E V(0.5)$ & 30 & 34 & 31 & 38 & 32 & 41 & 33 & 34 & 32 & 34 \\
\hline$E V(1.5)$ & 82 & 82 & 83 & 87 & 84 & 90 & 86 & 87 & 80 & 87 \\
\hline$D L(1)$ & 73 & 68 & 73 & 64 & 71 & 60 & 70 & 68 & 69 & 65 \\
\hline$D L(1.5)$ & $100 *$ & $100 *$ & $100 *$ & $100 *$ & $100 *$ & 99 & 99 & 99 & $100 *$ & 99 \\
\hline Av. & 75.6 & 76.7 & 76.2 & $77.6 *$ & 76.4 & 77.1 & 76.4 & 76.0 & 75.4 & 75.3 \\
\hline
\end{tabular}




\begin{tabular}{|c|c|c|c|c|c|c|c|c|c|}
\hline$k$ & & & & & 6 & & & & \\
\hline Tests & $\hat{T}_{n ; c_{2}}^{(r, k)}$ & $\hat{T}_{n}^{(r, k)}$ & $\hat{T}_{n}^{(r, k)}$ & $\hat{T}_{n ; c}^{(r, k)}$ & $\hat{T}_{n ; c_{3}}^{(r, k)}$ & $\hat{T}_{n ; c}^{(r, k)}$ & $\begin{array}{c}\hat{T}_{n ; c 3}^{(r, k)} \\
\end{array}$ & $\hat{T}_{n ; c 3}^{(r, k)}$ & $\hat{T}_{n ; c}^{(r, k)}$ \\
\hline Alt $\backslash r$ & 0.1 & 0.3 & 0.5 & 0.75 & 0.9 & 1.0 & 1.2 & 1.5 & 1.7 \\
\hline$W(0.8)$ & 34 & 29 & 26 & 33 & 33 & 33 & 31 & 30 & 31 \\
\hline$W(1.4)$ & $87 *$ & 81 & 80 & 84 & 84 & 84 & 83 & 82 & 82 \\
\hline$G(0.4)$ & 96 & 99 & 98 & 98 & 98 & 98 & 97 & 96 & 95 \\
\hline$G(2)$ & $97 *$ & 95 & 95 & 96 & 96 & 96 & 95 & 94 & 93 \\
\hline$L N(0.8)$ & 82 & 93 & 92 & 85 & 83 & 81 & 77 & 70 & 66 \\
\hline$L N(1.5)$ & 86 & 79 & 78 & 83 & 84 & 85 & 86 & 88 & 89 \\
\hline$H N$ & 55 & 49 & 50 & 50 & 52 & 53 & 54 & 55 & 57 \\
\hline$U$ & 96 & 97 & 97 & 92 & 94 & 94 & 95 & 97 & 97 \\
\hline $\mathrm{CH}(0.5)$ & 89 & 94 & 91 & 94 & 93 & 93 & 91 & 88 & 87 \\
\hline$C H(1)$ & 39 & 33 & 34 & 35 & 36 & 37 & 38 & 40 & 41 \\
\hline $\mathrm{CH}(1.5)$ & $100 *$ & $100 *$ & $100 *$ & $100 *$ & $100 *$ & $100 *$ & $100 *$ & $100 *$ & $100 *$ \\
\hline$L F(2)$ & 71 & 65 & 65 & 66 & 68 & 69 & 70 & 71 & 72 \\
\hline$L F(4)$ & 87 & 84 & 84 & 84 & 85 & 86 & 87 & 88 & 88 \\
\hline$E V(0.5)$ & 39 & 33 & 34 & 35 & 37 & 37 & 38 & 40 & 41 \\
\hline$E V(1.5)$ & 87 & 85 & 86 & 82 & 83 & 85 & 86 & 88 & 89 \\
\hline$D L(1)$ & 68 & 71 & 70 & 69 & 67 & 66 & 63 & 58 & 55 \\
\hline$D L(1.5)$ & $100 *$ & $100 *$ & 99 & $100 *$ & $100 *$ & 99 & 99 & 99 & 99 \\
\hline Av. & 77.2 & 75.6 & 75.3 & 75.7 & 76.0 & 76.3 & 76.0 & 75.5 & 75.4 \\
\hline
\end{tabular}

Table 5. Tests with Av. powers $<75.2$ and greatest powers for some alternatives; $n=50$

\begin{tabular}{|c|c|c|c|c|c|c|c|c|}
\hline$k$ & \multicolumn{2}{|c|}{1} & 3 & \multicolumn{5}{|c|}{6} \\
\hline Tests & $\hat{T}_{n ; c_{2}}^{(r, k)}$ & $\hat{T}_{n ; c 3}^{(r, k)}$ & $\hat{T}_{n ; c_{3}}^{(r, k)}$ & $\hat{T}_{n}^{(r, k)}$ & $\hat{T}_{n ; c_{2}}^{(r, k)}$ & $\hat{T}_{n ; c}^{(r, k)}$ & $\hat{T}_{n ; c}^{(r, k)}$ & $\hat{T}_{n ; c}^{(r, k)}$ \\
\hline Alt $\backslash r$ & -0.9 & -0.9 & -0.5 & -0.5 & -0.5 & -0.5 & 0.9 & 1.0 \\
\hline$W(0.8)$ & $57 *$ & $57 *$ & 51 & 41 & 46 & 43 & 17 & 16 \\
\hline$W(1.4)$ & 79 & 79 & 81 & 78 & 83 & 75 & 84 & 83 \\
\hline$G(0.4)$ & $100 *$ & $100 *$ & $100 *$ & $100 *$ & $100 *$ & $100 *$ & 16 & 11 \\
\hline$G(2)$ & 96 & 96 & 96 & 95 & $97^{*}$ & 96 & 94 & 93 \\
\hline$L N(0.8)$ & 77 & 76 & 86 & $97^{*}$ & 92 & $97^{*}$ & 61 & 58 \\
\hline$L N(1.5)$ & 89 & 89 & 86 & 81 & 77 & 59 & 59 & 53 \\
\hline$H N$ & 37 & 38 & 41 & 43 & 42 & 30 & $62 *$ & 61 \\
\hline$U$ & 83 & 83 & 87 & 93 & 84 & 65 & 99 & 99 \\
\hline $\mathrm{CH}(0.5)$ & $99 *$ & $99 *$ & $99 *$ & 98 & $99 *$ & $99 *$ & 17 & 13 \\
\hline $\mathrm{CH}(1)$ & 25 & 25 & 27 & 29 & 28 & 20 & $46^{*}$ & $46^{*}$ \\
\hline $\mathrm{CH}(1.5)$ & 99 & 99 & $100 *$ & $100 *$ & $100 *$ & 98 & $100 *$ & $100 *$ \\
\hline$L F(2)$ & 53 & 53 & 57 & 59 & 57 & 44 & $76 *$ & $76^{*}$ \\
\hline$L F(4)$ & 73 & 73 & 77 & 78 & 76 & 63 & $91 *$ & $91 *$ \\
\hline$E V(0.5)$ & 25 & 25 & 27 & 29 & 28 & 20 & $46^{*}$ & $46^{*}$ \\
\hline$E V(1.5)$ & 70 & 70 & 74 & 79 & 72 & 55 & 93 & 93 \\
\hline$D L(1)$ & 61 & 61 & 68 & 73 & 75 & $78^{*}$ & 51 & 48 \\
\hline$D L(1.5)$ & $100^{*}$ & $100^{*}$ & $100 *$ & $100^{*}$ & $100^{*}$ & $100 *$ & 99 & 98 \\
\hline Av. & 71.9 & 72.0 & 73.9 & 74.8 & 73.9 & 67.1 & 65.2 & 63.8 \\
\hline
\end{tabular}

Our simplest omnibus tests when $n=20$ and $n=50$ are

$$
\begin{aligned}
& \hat{T}_{n ; c_{2}}^{(-0.5,5)}=\frac{1}{\Delta_{n 1}^{(-1 / 2,5)} a_{n 1}^{(-1 / 2,5)}}\left[a_{n 1}^{(-1 / 2,5)} \frac{1}{\left(\begin{array}{c}
n \\
5
\end{array}\right)} \sum_{i=1}^{n-4}\left(\begin{array}{c}
n-i \\
4
\end{array}\right)\left(\frac{X_{i: n}}{\bar{X}_{n}}\right)^{1 / 2}\right. \\
& \left.-b_{n 1}^{(-1 / 2,5)} \frac{1}{n} \sum_{i=1}^{n-4}\left(\frac{X_{i}}{\bar{X}_{n}}\right)^{3 / 2} e^{-4 X_{i} / \bar{X}_{n}}-\frac{\sqrt{\pi}}{100 \sqrt{5}}\left(50 a_{n 1}^{(-1 / 2,5)}-3 b_{n 1}^{(-1 / 2,5)}\right)\right]^{2},
\end{aligned}
$$

where

$$
\begin{aligned}
& a_{n 1}^{(-1 / 2,5)}=\frac{3}{n}\left[\frac{2}{6561}-\frac{3 \pi}{40000}\right], \\
& b_{n 1}^{(-1 / 2,5)}=\frac{1}{n}\left[\frac{3}{5 \sqrt{5}} B_{\frac{4}{9}}\left(\frac{3}{2}, \frac{5}{2}\right)+\frac{10}{729}-\frac{57 \pi}{4000}\right],
\end{aligned}
$$




$$
\begin{aligned}
c_{n 1}^{(-1 / 2,5)}= & \frac{1}{\left(\begin{array}{l}
n \\
5
\end{array}\right)} \sum_{j=1}^{4}\left(\begin{array}{l}
5 \\
j
\end{array}\right)\left(\begin{array}{c}
n-5 \\
5-j
\end{array}\right)\left[\frac{4}{\sqrt{5(5-j)}} B_{\frac{5-j}{10-j}}\left(\frac{3}{2}, \frac{3}{2}\right)+\frac{j}{(10-j)^{2}}-\frac{\pi}{20}\right] \\
& +\frac{1}{5\left(\begin{array}{l}
n \\
5
\end{array}\right)}-\frac{\pi}{20}\left(\frac{1}{\left(\begin{array}{l}
n \\
5
\end{array}\right)}+\frac{1}{4 n}\right), \\
\Delta_{n 1}^{(-1 / 2,5)}= & a_{n 1}^{(-1 / 2,5)} c_{n 1}^{(-1 / 2,5)}-\left(b_{n 1}^{(-1 / 2,5)}\right)^{2}
\end{aligned}
$$

and

$$
\hat{T}_{n ; c_{3}}^{(0.5,4)}=\frac{1}{c_{n 1}^{(1 / 2,4)}}\left[\frac{1}{\left(\begin{array}{c}
n \\
4
\end{array}\right)} \sum_{i=1}^{n-3}\left(\begin{array}{c}
n-i \\
3
\end{array}\right)\left(\frac{X_{i: n}}{\bar{X}_{n}}\right)^{3 / 2}-\frac{3 \sqrt{\pi}}{32}\right]^{2}
$$

where

$$
\begin{aligned}
c_{n 1}^{(1 / 2,4)}= & \frac{3}{\left(\begin{array}{c}
n \\
4
\end{array}\right)} \sum_{j=1}^{3}\left(\begin{array}{l}
4 \\
j
\end{array}\right)\left(\begin{array}{l}
n-4 \\
4-j
\end{array}\right)\left[\frac{2}{(4-j) \sqrt{4-j}} B_{\frac{4-j}{8-j}}\left(\frac{5}{2}, \frac{5}{2}\right)+\frac{2 j}{(8-j)^{4}}-\frac{3 \pi}{1024}\right] \\
& +\frac{3}{32}\left[\frac{1}{\left(\begin{array}{c}
n \\
4
\end{array}\right)}-\frac{3 \pi}{32}\left(\frac{1}{\left(\begin{array}{c}
n \\
4
\end{array}\right)}+\frac{9}{4 n}\right)\right]
\end{aligned}
$$

with Av. powers 47.2 and 46.5 for $n=20$, and 76.7 and 76.4 for $n=50$, respectively.

Our most powerful test for $n=20$ is $\hat{T}_{n ; c_{2}}^{(-0.5,5)}$ with Av. power 47.2 , and for $n=50$ is $\hat{T}_{n ; c_{2}}^{(-0.3,5)}$ with Av. power 77.6.

Acknowledgments. I am very grateful to my friend Kerwin Morris for corrections and suggestions given in preparing versions and to Ms. Anna Nosalewicz for simulations and typing.

\section{References}

[1] G. E. Andrews, R. Askey and R. Roy, Special Functions, Cambridge University Press, 2000.

[2] L. Baringhaus and N. Henze, A test uniformity with unknown limits based on D'Agostino's D, Statist. Probab. Lett. 9 (4) (1990), 299-304.

[3] M. Bieniek and D. Szynal, Recurrence relations for distribution functions and moments of kth record values, J. Math. Sci. 111 (3) (2002), 3511-3519.

[4] M. Bieniek and D. Szynal, Limit distributions of differences and quotients of non-adjacent kth record values, Probab. Math. Statist. 23 (1) (2003), 19-38.

[5] M. Bieniek and D. Szynal, On $k$-th record times, record values and their moments, J. Statist. Plann. Inference 137 (2007), 12-22.

[6] A. Cabaña and E. M. Cabaña, Goodness-of-fit to the exponential distribution, focused on Weibull alternatives, Commun. Statist. Simul. Comput. 34 (2005), 711-723.

[7] M. Csörgó, V. Seshadri and M. Yalovsky, Applications of characterizations in the area of goodness of fit, in: C. P. Patil et al. (eds.), Statistical Distribution in Scientific Work, Vol. 2, Reidel, Dordrecht, 1975, 79-90.

[8] W. Dziubdziela and B. Kopociński, Limiting properties of the $k$-th record values, Zastos. Mat. 5 (2) (1976), 187-190. 
[9] Z. Grudzień and D. Szynal, Characterization of continuous distributions via moments of record values, J. Appl. Statist. Sci. 9 (2) (2000), 93-104.

[10] N. Henze and S. G. Meintanis, Goodness-of-fit tests based on a new characterization of the exponential distribution, Commun. Stat. Theory Meth. 31 (2002), 1479-1497.

[11] N. Henze and S. G. Meintanis, Recent and classical tests for exponentiality: a partial review with comparisons, Metrika 61 (2005), 29-45.

[12] T. P. Hill and V. Perez-Abreu, Extreme-value moment goodness-of-fit tests, Ann. Inst. Statist. Math. 53 (3) (2001), 543-551.

[13] A. J. Lee, U-Statistics, Theory and Practice, Marcel Dekker, New York, 1990.

[14] G. D. Lin, Characterizations of continuous distributions via expected values of two functions of order statistics, Sankhyā Ser. A 52 (1990), 84-90.

[15] I. Malinowska, K. Morris and D. Szynal, On dual characterizations of continuous distributions in terms of expected values of two functions of order statistics and record values, J. Math. Sci. 121 (5) (2004), 2664-2673.

[16] K. W. Morris and D. Szynal, Goodness-of-fit tests, based on characterizations in terms of moments of order statistics, Appl. Math. 29 (3) (2002), 251-283 .

[17] K. Morris and D. Szynal, Tests derived from characterizations in terms of moments of record values, Appl. Math. 30 (1) (2003), 11-37.

[18] K. Morris and D. Szynal, Goodness-of-fit tests using dual versions of characterizations via moments of record values, J. Math. Sci. 122 (4) (2004), 3384-3403.

[19] K. Morris and D. Szynal, Tests resulting from characterizations using record values, J. Math. Sci. 131 (3) (2005), 5646-5656.

[20] K. Morris and D. Szynal, Goodness-of-fit tests via characterizations, IJPAM 23 (4) (2005), $491-555$.

[21] K. Morris and D. Szynal, Goodness-of-fit tests based on characterizations involving moments of order statistics, IJPAM 38 (1) (2007), 83-121.

[22] K. Morris and D. Szynal, Some U-statistics in goodness-of-fit tests derived from characterizations via record values, IJPAM 46 (4) (2008), 507-582.

[23] D. A. Pierce, The asymptotic effect of substituting estimators for parameters in certain types of statistics, Ann. Statist. 10 (2) (1982), 475-478.

[24] N. M. Temme, Special Functions. An Introduction to the Classical Functions of Mathematical Physics, John Wiley \& Sons, 1996.

[25] Y. H. Too and G. D. Lin, Characterizations of uniform and exponential distributions, Stat. Probab. Lett. 7 (5) (1989), 357-359. 
